

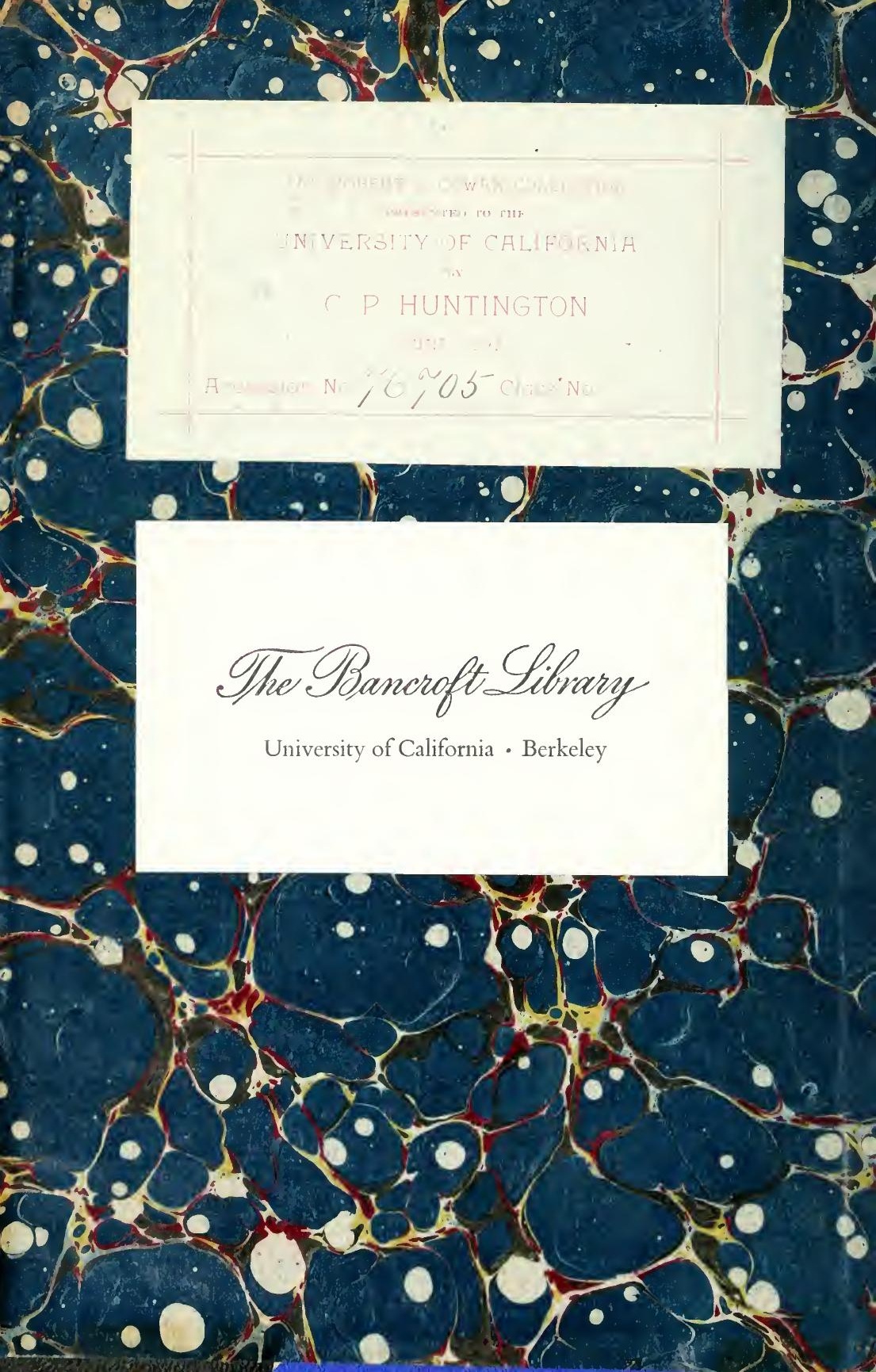


Digitized by the Internet Archive in 2008 with funding from Microsoft Corporation 




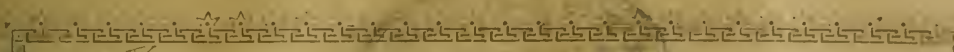

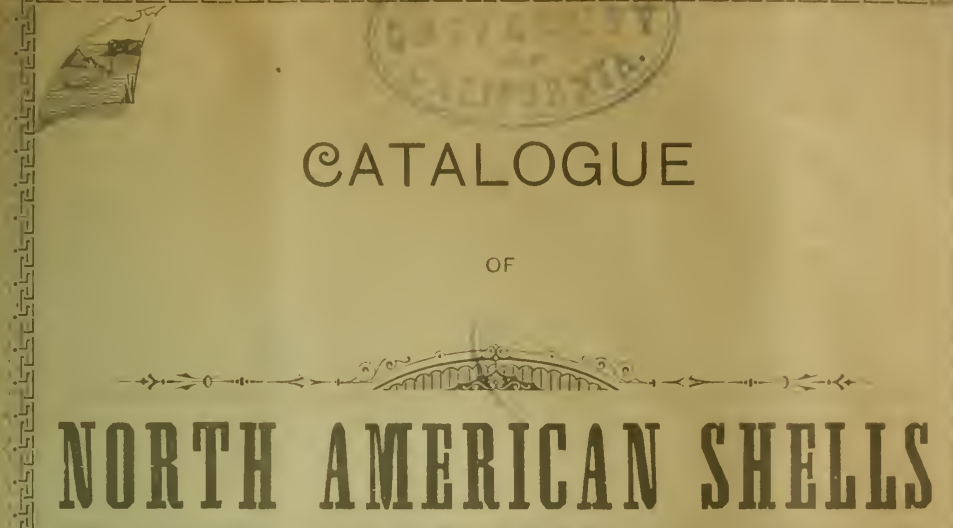

COLLECTED AND FOR SALE BY

\section{HENRY HEMPHILL,}

ana mincion.

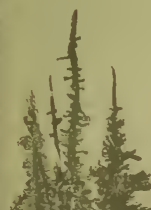

CAITHODTEIAL

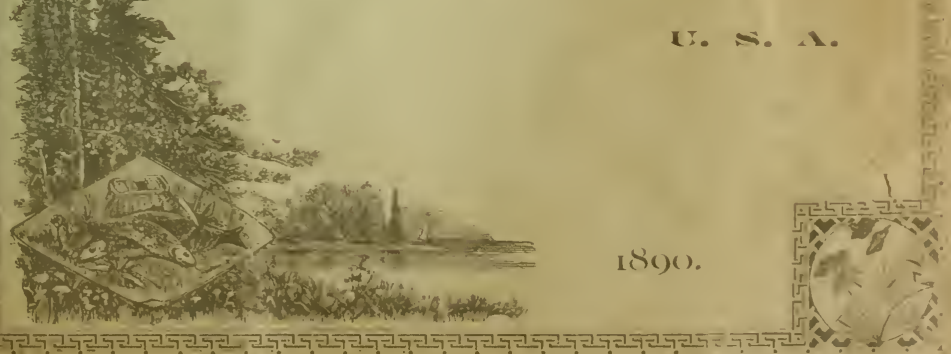




\section{NO'TE.}

The following list of shells is mare from my own personal collections, which probably embraces the largest number and best series of American shells that has ever been offered to the student for close scientific study. The large number of varieties offered add very much to the value of the series, but it must be understood that some of them do not represent very great differences from the preceding or the following variety or specie. The object being to show their relations to one another by a closely graded series of interniediate forms. In some in. stances I have not been able to do this, yet for the most part they are so arranged that their relationship can be easily inferred.

I have reduced many of the so-called species to varieties and am convinced that very many more should be treated in the same manner. Some conchologists may differ from me in this particular, but while I esteem their opinions highly I must be true to my own convictions and trust to further study to prove the truth of my position.

Next to the objects themselyes I regard variation as the most instructive and important part of the study of conchology, and when the student has become fairly familiar with it, he will find it possesses a charm rarely found in other fields of investigation. The Land Shells are largely arranged in groups, as suggested by the forni and sculpturing of the shells, and I have placed at the head of each group the species that seem to be the progenitor of those that follow.

In this arrangement of the land shells, very little attention has been given to genera or subgenera as I think it best to follow nature wherever she leads rather than to follow altogether the vagaries of systematists and parlor students. This arrangement may also serve, not only to attract attention to the relations existiug between the individuals of each group, but to the necessity of some more natural classification of these interesting little animals. I am strongly impressed that no system of classification can be devised that will be acceptable to a majority of Naturalists until the laws of variation are more thoroughly understood, for they are the key to the system.

The nomenclature has been brought down to the latest date, and every effort has been made to make the series as scientifically correct as possible, and especial attention is invited to the fact that all the shells listed on the following pages have been collected by myself personally, and have not been picked up by travellers, or bought from irresponsible persons, so that the localities given with each specie or variety can be relied upon as strictly correct.

HFNRI HEMPHILI. 


\section{HEMPHILL'S CATALOGUE}

\section{() $\mathrm{H}$ \\ North American Shells}

\section{CLASS CEPHALOPODA.}

FAMILY OCTOPODIDE. GENUS OCTOPUS.

1 2

O. punctatus. Gabb.. California........\$ 50 FAMILY SPIRULIDA.

3 S. Peroni, Lam. Florida

\section{CLASS GASTROPODA. FAMILY MURICIDA. GENUS MUREX. Subgenus Chicoreus.}

4 M. rufus, Lam. Florida Subgenus Phyllonotus.

5 M. Pomum, Gmel. Florida.. Subgenus Pteronotus.

6 M. festivus, Hds. California.

7 M. Californicus, Hds. Lower Cal.....

8 M. trialatus, Sowb. GENUS CFROSTOMA.

C. foliatum, Gmel. California

C. Nuttalli, Conr. California.

C. " var. broad banded

C. "

var. narrow white

var. " banded...

var. " dark

var. monoceros. Low.Cal..

GENUS EUPLEURA.

E. caudata, Say. Florida.

var, darker

GENUS OCINEBRA.

O. incisus, Brod. California

O. alveatus, Kiener. Florida

O. nuceus, Morch. Florida.

O. Poulsonii, Nutt. California........

O. Lurida, Midd. California.

O. "var. "

O. "var. "

O. "var."

O. "var."

O. circumtexta, Stearns. California...

O. " var. " " ...

O. interfossa, Cpr. California ..........

0 " var. " "

O. " var, " " . ".......

O. gracillima, Stearns. California.....
GFNUS MURICIDA:.

34 M. multangula, Philippi. Florida....\$ 15

35 M. Floridana, Conr. Florida.......... 10

36 M. " var. " " ........ I0

GFNUS UROSALPINX.

37 U. cinereus, Say. Florida

38 U. perrugatus, Conr.

39 U. " small. “"

40 U. Tampaensis, Conr.

41 II. " var.

IO

FAMILY PURPURIDA.

GFNUS PURPURA.

42 P. deltoidea, Lam. Florida............. I5

43 P. hamastoma, Linn. Lower Cal. ... 25

44 P. "var, smooth. " " $\ldots 25$

45 P. " "var. undata, Lam. Fla... I5

46 P. " var. Floridana, Conr. Fla. I5

Crispy-foliated forms.

47 P. crispata, Chem., dark. Puget Sound. 25

48 P. " var. banded. " " 25

49 P. " var. white, " " 25

25

Foliations becoming obsolete.

5o P. crispata, var. chocolate. Cal........ 15

5 I P. "var. lemon-color. Cal... I5

Sinooth forms.

52 P. crispata, var, banded. Puget Sound.

54 P. “ var. septentrionalis. Rve. Cal.

Dark. Cal.

“var. var. Banded Cal.

57 P. lima, Marty'u, dark. Cal............. 58 P. " var, white. Cal.

59 P. " var. small.

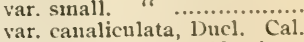

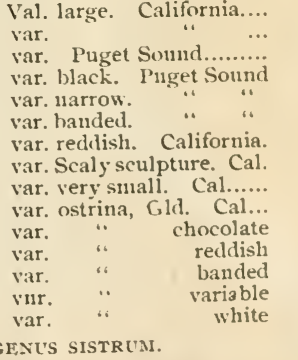


GENUS MONOCEROS.

78 M. lugubre, Sowb. large., Lower Cal.\$o 20

79 M. " var. stumpy. " 20 " 120

80 M. " " var. small.

8 I M. " var.

82 M. " var.

83 M. engonatum, Conr. Cal. \& L. Cal.

$84 \mathrm{MI}$.

$85 \mathrm{M}$.

$86 \mathrm{M}$.

$87 \mathrm{M}$.

$88 \mathrm{MI}$.

"6

var. Lower Cal ......

var. intermediate...

var. spiratum, Blainv

var.

var. small ............

89 M. engonatum, var. lapilloides. Conr. Cal...

90 M. engonatum, var. lapilloides, globose. Lower California.........

9 I M. engonatum, var. paucilirata

Stearns. Cal...

92 M. engonatum, var. paucilirata, typical, Cal.

GENUS CHORUS.

93 C. Belcheri, Hds. California

\section{FAMILY TRITONIDA: \\ GENUS TRITON.}

$94 \mathrm{~T}$. femorale, Linn

GENUS RANELIA.

95 R. Californica. California.

FAMILY FUSIDAE.

GENUS FUSUS.

96. F. Kobelti, Dall. California

SUBFAMILY FASCIOLARIINA.

GENUS FASCIOLARIA.

97 F. tulipa, Linn. Florida

$98 \mathrm{~F}$. " var. "

$99 \mathrm{~F}$. distans, Lam. " " $\quad$ ".............. 25

IOO F. " var. "

IOI F. gigantea, Kiener. " $\quad \ldots . . . \ldots \ldots . . .$. I 35

IO2 F. " var. like princeps, Fla.. I 50 GENUS LATIRUS.

I03 L. Coyhuesonicus, Sowb. Florida ... 50 GENUS LEUCOZONIA.

I04 L. cingulifera, Lam. Florida..

FAMILY BUCCINIDE.

GENUS MELONGENA.

I05 M. corona, Gmel.incurved spines. Fla.

106 M. " var. double row of spines.

Florida

I07 M.

var. Florida..................

GENUS FULGAR.

I08 F. perversus, Linn., very large. Fla... I oG

$109 \mathrm{~F}$. " var. Fla................... I 0

I IO F. pyrum, Dillw.

GFNUS FUTHRIA.

I 1 E. dira, Rve. California.

GENUS CANTHARUS.

112 Cantharus, tincta. Conr. Florida.......

var.

var.

var.

var.

var.
IIS Cantharus elegans, Gray. Iower Cal...\$0 25

II9 " lugubis. C. B. Ad. " " ... 25

120 " cancellaria, Conr.

GENUS MACRON.

I 2 I M. athiops, Rve. deep grooves. I. Cal. 50

122 M. Kelletti, Fhs, smooth. Lower Cal. 50

123 M. lividus, A. Ad. Cal. \& Lower Cal.. Io

G.NIS PHOS.

124 P. parvis, C. B. Ad. Florida

FAMILY NASSIDA.

GENUS NASSA.

IO I $25 \mathrm{~N}$. vihex, Say., large. Florida.......... 5

$10126 \mathrm{~N}$. " var. medium. " " ......... 5

I 27 N. " var. small. " " ....... क

I28 N. " var. dark. " " "....... \$

$15129 \mathrm{~N} . \quad$ " var, white. “ $\ldots$........ 5

$130 \mathrm{~N}$. " var. " small. " ......... 5

I3I N. " var. tegula, Rve. Cal. 5

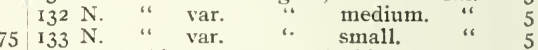

I34 N. anibigua, Mont. Florida.......... Io

I35 N. “ var. " $\quad$ ".......... 15

I36 N. fossata, Gld. large. California....... I5

I37 N. " var. small. " " ...... I5

I38 N. perpinguis, Hds. "

139 N. mendica, Gld. "f ....... Io

I40 N. " var. Cooperi, Cpr. Cal.... Io

I4I N. obsoleta, Say. Florida............... 5

FAMILY TURBINELIIDA:.

GENUS VASUM.

142 V. muricatnnı, Born. Florida........ 50

\section{FAMILY MITRIDE:}

GENUS MITRA.

I43 M. maura, Swains. Lower Cal...... 25

I44 M. microzonias, Lam. Florida........ I5

I45 M. Floridana, Dall. " "..... 25

I 46 M. albocinctus, C. B. Ad. " ...... 15

147 M. Hanleyi, Dohn. " ...... I5

\section{FAMII, MARGINFLLIDA.}

\author{
GENUS ERATO.
}

20 I 48 F. vitellina, Hds. Califormia ....

I49 ki Columbella Mke "

GFNUS MARGINEILLA.

50 I50 M. opalina, Stearms. Florida......... 25

I51 M. aureocincta, " "

50 I52 M. carnea, Storer. “ “ $\quad$ “........ 25

5o I53 M. apicina, Mke, …..... 5

I54 M. " var. "

155 M. " var. virginea Jouss. Fla.. 10

I56 M. Jewetti, C'pr. California. ........... 5

I57 M. regularis, Cpr. " " $\quad \ldots \ldots \ldots \ldots . .6$

15 M. " " var.

159 M. nisiuta, P'fr. Florirla .............. 5

160 M. lacrinula, Gid. " ............. 5

I6I M. pyriformis, C’pr. California........ 5 Subgenus Persecula.

I5 I62 M. Sagatata, His. Florida............ Io

15
15

I5 I63 M. snccinea, Conr. Florirla............ 5

15 164 M. " 16 var. "

15 165 M. varia, Sby. Califormia................ 5 


\section{FAMILY OLIVIDAE. GENUS OLIVELLA.}

I66 O. mutica, say. Florida.

1670 . " var.

I68 O. " var.

I69 O. jaspidea, Gmel.

r7o O. floralia, Duclos. "

I7I O. boetica, Cpr. California

1720 . " var.

GENUS OLIVA.

173 O. arênosa, var. Lam. Lower Cal...

I74 O. litterata, Lam. Florida

Subgenus Callinax.

I75 O. biplicata, Sby. California

$176 \mathrm{O}$.

$177 \mathrm{O}$.

var.

var.

\section{FAMILY COLUMBEILIIDA:}

\section{GENUS COLUMBELIA}

178 C. fuscata, Sowb. Lower California...

179 C. mercatoria, Linn. Flurida.

$180 \mathrm{C}$.

I 8 I C.

$182 \mathrm{C}$.

$183 \mathrm{C}$. rustica Lin

I84 C. " var.

I85 C. humerosa, Cpr. Lower California Section Alia.

IS6 C. carinata, Hds. California.

I87 C. " var. Hindsii, Rve

I88 C. " " var. gausapata, Gld

I89 C. " " var. California, Cpr

I90 C. " var. Gouldii, Cpr.

$191 \mathrm{C}$.

var.

$192 \mathrm{C}$.

var.

\section{Section Mitrella.}

I93 C. cribraria, Lam. Florida.

I94 C. " var. small. Florida....

I95 C. cervinetti, Cpr. Lower Califoruia

I96 C. luuata, Say. Florida................

I97 C. aurantiaca, Dall. California

198 C. tuberosa, Cpr.

I99 C. " var.

200 C. chrysalloidea, Cpr.

Section Anachis.

201 C. coronata, Sowb. Lower California 202 C. acuta, Stearus. Fla.

203 C. avara, Say. Fla.

20.4 C. " var, semiplicata, Stearns......... 205 C. ornatus, Lam. Fla....

206 C. " var.

207 C. " var

208 C. pulchella, Kiener. Fla..

209 C. " var. Florida

$2 I \mathrm{IC}$

var.

var.

\section{Section Seminella.}

212 C. pulchrior, A. Ad. Lower Cal ...... $2 I_{3}$ C. tincta, Cpr. Lower Cal.

2 I 4 C. Gaskoini, Cpr.

2 I5 C. ostreicola, Melville. Fla ..............

216 C. penicellata, Cpr. Cal.

217 C. subturrita, Cpr.

GENU'S ESOPUS.

2 I8 F. Hemphilli, Stearns. I.ower Cal

GENUS ENGINA.

2 Ig F. turbinella, Kiener. Fla............ \& 15

GENUS AMPHISSA.

220 A. corrugata, Rve. Cal............... 5

$221 \mathrm{~A}$. " var. versicolor, Dall. Cal

$222 \mathrm{~A}$.

$223 \mathrm{~A}$.

224 A.

$225 \mathrm{~A}$.

25

20

226 C. Californicus, Hds. California .....

227 C. verrucosus, Hwass. Florida .........

228 C. proteus, Hwass.

229 C. Florilanus, Gabb.

230 C. Pealei, Green.

\section{FAMILY PLELROTOMIIDE.}

GFNL:S DRILLIA.

10

5

5

5

5

5

5

5

5

5

6245 M. balteata, Rve Florida....

246 M. biconica, C. B. Ad. ."

5247 M. plicosa, C. B. Ad.

$524 \mathrm{~S}$ M. stellata, Stearns.

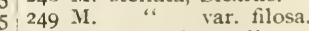

5250 M. atrostyla, Dall.

5 25I M. limonitella, Dall.

5.252 MI. cerinella, Dall.

5253 M. melanitica, Dali.

10 254 M. merita, Gld. Califormia.

Section Citharu.

5. 255 M. neglecta, A. Ail. I.ower California

$5256 \mathrm{MI}$. angulata, Cpr.

5257 M. densistriata, Cor.

5

5

5

5

5

5

5

26r ' 1 . ". var.

262 '. ". var.

5.

\section{$369 \mathrm{~T}$. albociacta, Cpr.}

270 T. ." var.

\section{0}

IO

50

10

\section{I0}

10

10

\section{5}

15

15

15 


\section{FAMILY CANCELLARIIDAE. GENUS CANCELLARIA.}

272 C. reticulata, Linn. Florida

\section{FAMILY STROMBIDA.}

273 S. gigas, Linn. Florida.

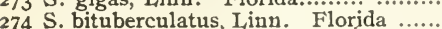
$275 \mathrm{~S}$. var. small. " ...... 276 S. pugilis var. alatus, Gmel.

277 S. " var, nodules obsolete. Fla..

FAMILY CYPRAIDAE. GENUS CYPRFA.

278 C. exanthema, Linn. Florida. 279 C. cervus, Linn.

280 C. spadicea, Gray. Califoruia............. 281 C, sowerbyi, " Lower Cal ............ 282 C, cinerea, Linn, Florida .............. GENUS TRIVIA.

283 T. solandri, Gray, Lower California... 284 T. " var.

285 'T, Californica, Gray, California ...... 286 'T, quadripunctata, Gray, Florida...... FAMILY OVULIDA: GENUS OVULA, Subgenus Cyphoma.

287 O. gibbosa, Linn. Florida. Subgenus Neosimnia. 288 O, variabilis, C. B. Ad. Lower Cal...... FAMILY DOLIIDAE, GENUS PYRULA.

289 P. papyratia, Say. Florida.

\section{FAMILY NATICIDAE.} GENUS NATICA.

290 N. canrena, Linn, Florida.

29 I N “ var " . . 292 N, morochiensis, Gmel, Lower Cal... 293 N. sp, small, Florida.

\section{Subgenus Neverita.}

294 N, duplicta, Say, Florida.............. 295 N. Recluziana, Petit, California......... Section Lunatia.

296 N. Lewisii, Gld, Puget Sound Section Mamma,

297 M. uber, Val. Lower California

GENUS SIGARITUS,

298 S, perspectivus, Say. Florida.

GENUS LAMELLARIA

299 L, Diegoensis, Dall, California. 300 L. Stearnsii, Dall.

GFNUS VF:LUTINA.

3or V. lævigata, Linn. California.

\section{FAMIIY CALYPTR AEIDXE.}

GENUS CRUCIBULUM.

302 C, scutellatum, Gray Lower Cal...... 303 C, " var,tubiferum, Linn, L.C. $304 \mathrm{C}$, $305 \mathrm{C}$. var, var.

\section{GFNUS CREPIDUTA}

307 C, fornicata, Say, Florida

308 C. ollyx, var, rugosa, Nutt. Califormia

C.

3 Io C. arenosa, Brod. Lower California... Io

31 I C. dorsata, var. lingulata, Gld. Cal.... 5 Section Janicus.

312 C. unguiformis, Say. Florida. .......... 313 C. " var. navicelloides, Nutt. California................. Section Crypta.

314 C. aculeata, Gmel. Florida.............. 5

3I5 C. " var. " " $\quad \ldots \ldots \ldots \ldots .65$

$316 \mathrm{C}$.

317 C. " " var. "

3IS C. " var. California .......... I0

FAMILY CAPULIDA.

GINUS HIPPONYX.

319 H. antiquatus, Linn. Florida \& Cal.. 5 $320 \mathrm{H}$. barbatus, Sowb. Lower Cal........ 5 321 H. tumens, Cpr. California............ Io FAMILY VERMETIDÆ.

CFNUS VERMFTUS.

322 V. aunellum, Morch. California ...... I5 o 323 V. squamigerus' Cpr. “ $\quad \ldots . . .15$ 324 V. eburneus, Rve. Lower “ $\ldots . .$. I5 325 V. Hindsii, Gray. $\quad$ “ $\quad \ldots \ldots$. I5 326 V. lituella, Morch. California......... I5 GENUS VERMICULARIA.

327 V. lumbricalis, var.radiculus, Stimpson Florida......................... 20 $328 \mathrm{~V}$. lumbricalis, var. radiculus. small... 20 329 V. " var. " white... 20 330 V. nigrescens, Dall. Florida........... 20 $331 \mathrm{~V} . \quad$ sp. “ $\quad \ldots . . . . \quad 15$ $332 \mathrm{~V} . \quad$ sp. " $\quad$........ 15 333 V. $\quad$ sp. Cal........... 15 334 V. sp. “ $\ldots \ldots \ldots$. I5

FAMILY TURRITFLIIDA:

GENUS TURRITELI,A.

335 T. cooperi, Cpr. California. Subgenus Mesalia.

336 'T. acicula, Stimpson. Cal............. I0 FAMII, CAECIDE. GINTS CACUM.

Io 337 C. glubrum, Mont. Cal............... $33^{8}$ C. glabriformis, Cpr. 339 C. Orcutti, Dall.

1 regard the above as different forms of one species. Section Annulata.

20340 C. Flordanum, stimpson. Florida.... 34 I C. firmatum, California ................... 342 C. crebricinctum, Cpr. Cal.

343 C. Californica, Dall. Section Costutata. 5

. 5

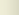

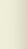

.

.




\section{FAMILY EULIMIDA: GENUS EULIMA.}

347 E. micaus, Cpr. California 348 E. " var. Lower " 349 E. conoidea, Kust and Stimpson. Fla... 350 E. subcarinata, D'Orb. Lower Cal..... Subgenus Liostraca.

35 I E. rutila, Cpr. Cal..

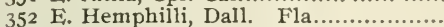

353 E. conica, C. B, Ad. Lower Cal.

354 E. solitaria, C. B. Ad.

\section{FAMILY PYRAMIDELLIDA:。 GENUS PYRAMIDELLA,}

355 P. conica, C. B. Ad. Lower Cal. $356 \mathrm{P}$.

var $\mathrm{Fla}$

GENUS OSCILI,A.

357 O. nivea, Morch. Florida.

$35^{8}$ O. insculpta, Cpr. Cal.

359 O, exarata, Cpr.

\section{Subgenus Chrysallida.}

360 C. communis, C. B. Ad. Lower Cal... 36I C. pumila, Dall, Lower Cal........... $362 \mathrm{C}$.

\section{Section Mumiola}

363 C. cincta, Cpr. California.

364 C. anianta, Dall.

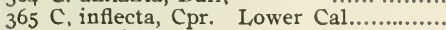
366 C. turricula, De'Folin.

\section{FAMILY TURBONILLIDE.}

GENUS TURBONILLA.

367 T. tenuicula, Gld. California $368 \mathrm{~T}$.

$369 \mathrm{~T}$

var.

$270 \mathrm{~T}$. aurantiaca, Cpr. Lower Cal

37 I 'T: castaneus, Cpr. Cal.

$372 \mathrm{~T}$. torquata, Gld.

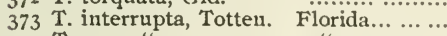
$374 \mathrm{~T}$

375 T. virga, Dall.

376 T. punicea, Dall.

$377 \%$.

$378 \mathrm{~s}$.

“ $\quad$ sp.

Subgenus Dunkeria.

379 D. laminata, Cpr. California 3 So $\mathrm{D}$. 38 I D.

var.

var.

Subgenus Parthenia.

${ }_{3} \mathrm{~S}_{2} \mathrm{P}$. cedrosa, Dall. Florida

GENUS ODOSTOMIA.

${ }_{3} \mathrm{~S}_{3}$ O. satura, Cpr. California.

${ }_{3} \mathrm{~S}_{4} \mathrm{O}$. gravada, Cpr.

3850 . " ? var.

386 O. straminea, Cpr.

$387 \mathrm{O}$. " var.

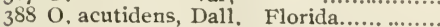

389 O. conoidea, Cpr. Lower Cal.............

390 O, impressa, Say. Fla.

39 I 0.

var. granatina, Dall. Fla

\section{Section Miralda.}

392 O. quinquecincta, Cpr. California...... Section Evalea.

393 O. tenuisculpta, Cpr. Cal.

394 O. " var. incisa, Cpr. Cal

395 O. graciliente, Cpr. Cal.

\section{FAMILY IANTHINIDA.}

GENUS IANTHINA.

$\$ 0 \quad 15$

I5

I 5

20

25

50

20

IO

IO
20

I5

20

20

25

20

20

\begin{tabular}{l|} 
I5 \\
25 \\
20 \\
20
\end{tabular}

IO

IO

IO

15

15
4IS C. " " var. "

396 I. communis, Lam. Fla.

397 I. globosa, Swains.

398 I. exiguus, Linn.

\section{HAMILY SCALARIIDA.}

GFINUS SCALARIA.

399 S. hexagona, Sowb. Lower Cal........ 25

400 S. Hindsii, Cpr. California................. 20

401 S. Indianorum, var. tincta, Cpr. Cal... I5

402 S. angulata, Say. Fla.................. 20

403 S. multistriata, Say. "

404 S. subcoronata, Cpr.

Subgenus Opalia.

405 O. crenatoides, Cpr. California.......... 20

IAMII,Y CFRITHIDA:.

GENUS CERITHIUM.

406 C. uncinatum ? Gmel. Florida........... Io

407 C. literatum, Born.

$408 \mathrm{C}$. " var. small " "........ 15

409 C. eburmeum, Brug. " " ........ IO

4 IO C. muscarum, Gmel. " " ......... IO

4 I I C. "

412 C. " " var. "

$4 \mathrm{I} 3$ C. $\quad$ ". $\quad$ var.

414 C. $\quad$ ". var. $\quad$ " $\quad$ ve........

415 C. " $\quad$ sp. "

416 C. interruptum, Mke. Lower California. Io

$4 \mathrm{I} 7 \mathrm{C}$. " " var. "

GENUS CERITHIDEA.

419 C. Montagnei, D'Orb. Lower Cal..... 20

420 C. Sacrata, Gld. "

42 I C. " " var. albonodosa, Cpr. L.C. Io

422 C. " var. pullata, Gld. Cal.... 10

423 C. "

424 C. " var. ".... 10

425 C. scalariformis, Say. Fla.............. Io

$426 \mathrm{C}$. " var. "

427 C. "

428 C. turrita, Stearns. " ".............. 10

429 C. " var. tenuis, Pfr " ............... IO

GENUS BITTIUM.

430 B. filosum, Gld, Califoruia............... 5

43 I B. " " var. ".

432 B. " " var.

433 B. " var. esuriens, Cpr......... 5

434 B. attenuatum, Cpr. California........ Io

435 B. " var.

436 13. " var.

437 B. $\quad$ var. " $\quad$ va ......... IO

$43^{8}$ B. armillatum, Cpr. $\quad$.. $\quad \ldots . . . . . . \quad 15$

439 B. subplanatum, Cpr. Lower Cal........ 15

440 B. quadrifilatum, Cpr. “...... 5

44 I B. nigrum, Totten. Florida............... 5

442 B. " var. "

443 B. " var. "

444 B. . var. " $\quad \ldots \ldots \ldots \ldots .65$

445 B. Cerithoides, Dall. Lower Cal....... I5

GENL'S CERITHIOPSIS.

446 C. tubercularis, Mont. Cal............. 5

447 C. $\quad$ " var. " ............ 5

448 C. " Cpr. Cal......................... 5

449 C. columna, Cpr. Cal................... Io

450 C. subulata, Mont. Florida............. Io

45 I C. metaxae, D'Orb. Lower Cal......... 20 
GFNUS CFRITHIOPSIS (Continned).

452 C. Greeni, C.B. Ad. Florida............ 453 C. neglecta, C. B. Ad. I.ower Cal...... 454 C. minimus, Bru. Florida........ $455 \mathrm{C}$. sp.

\section{Section Seila.}

456 C. assinilata, C. B. Ad. Lower Cal... 457 C. terebralis. C. B. Ad. Florida......... GENUS TRIFORIS.

455 T. wigrocinctus, C. B. Ad. Florida.... 459 T. adversa, Mont. Iower Cal.... 460 T. " var.

\section{FAMILY LITTORINIJF.} GFNUS LITTORINA.

46I I. rudis, var. sitchana, Phil. A. Puget Sound.............................

462 L. rudis, var. sitchana, Phil. B. Puget Sound..

463 I. rudis, var. sitchana, Phil. Puget Sound.

46.4 I. rudis var. sitchana, Phil. Priget Sound.

465 I. rudis var, sitchaua, Phil. Puget Sound.

466 L. rudis, var. sitchana, Phil. Puget Sound.

c.

D. .......

F.

467 I. rudis, var. sitchana, Ph11. G. Puget Sound...

468 L. rudis, var. sitchana, Phil. H. Puget Sound.

469 L. rudis var. sitchana, Phil. I. I.

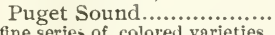
A fine series of colored varieties. Section Melarapha.

470 L. scabra, Linn. A. Florida.

$47 \mathrm{I} \mathrm{I}$

472 L.

473 L.

$474 \mathrm{~L}$.

475 L.

$\begin{array}{lll}\text { " var. } & \text { B. } \\ \text { " var. } & \text { C. } \\ \text { " var. } & \text { D. } \\ \text { " var. } & \text { E. } \\ & \text { var. } & \text { F. }\end{array}$

A series of colored varieties.

476 L. irrorata, Say. Florida.

477 L. " var.

478 L. varia, Brod. Lower Cal.

479 L. planaxis, Nutt. A. California.....

$480 \mathrm{~L}$. " " var. B. "

48 I L. " " var. C.

482 L. " " var. D.

483 L. " " var. F,

484 L. " " var. F.

385 L. " " var. G.

486 L. " " var. II.

487 L. " " var. I.

488 L.

Inother very fine series of colored varieties.

489 L. ziczac, Chem. Florida.................

490 L. scutulata, Gld. A. California......

491 I.

492 L.

$493 \mathrm{~L}$,

494 L.

$495 \mathrm{~L}$.

496 L.

497 L.

498 L.

499 L.

\begin{tabular}{l|ll}
5 & 500 & $\mathrm{~T}$ \\
$\mathrm{I}$ & $50 \mathrm{I}$ & $\mathrm{T}$.
\end{tabular}

GFNUS TECTARIUS.

(iENT'S MODULUS

502 M. Floridanus, Conr. Fla.............. 5

503 M. lenticulus, Chem. " $504 \mathrm{M}$.

var.

GENUS LACUNA.

Io $505 \mathrm{~L}$. unifasciata, Cpr. California......... Io

Io $506 \mathrm{~L}$. " " var.aurantiaca.Cpr. Cal I5

Io 507 L. porrecta, Cpr. Cal.................. 15 FAMILY FOSSARIDA.

GEINUS ISAPIS.

508 I. fenestrata, Cpr. California.......... I5

5509 I. obtusa, Cpr.

FAMIILY PLANAXIDAE.

GENUS PLANAXIS.

$55 \mathrm{lo}$ P. lineatus, Da'Casta. Fla.

51 I P. " " var. "

5 I I 2 P. * var.

Subgenus Diala.

55 I3 D. marmorea, Cpr. Cal.

FAMIIIY RISSOIDAE. GFNUS RISSOA:

Section Cingula.

5

5

5

5 5It

$517 \mathrm{R}$

Subgenus Alvania.

5

I 5

IO

I5

GF,NUS RISSOINA.

5 . 5 IS R. interfossa, Cpr. Cal................ I5

5 II9 R. " var. " 5 ............... I5

$5520 \mathrm{R}$. interclathrata. Cpr. MisS. Cal,..... 20

5 52I R. bryerea, Mont. Lower Cal.......... I5

5522 R. pulchra, C. B. Ad. Florida........ Io

5 523 R. dubiosa, C. B. Ad. “ ......... Io

524 R. decussata, Mont. " “ ......... Io

$5525 \mathrm{R}$. Browniana, D'Orb. “ ......... Io

Io $526 \mathrm{R}$. Chesneli, Mich. $\quad$ ".......... Io

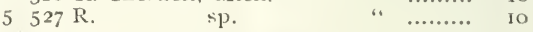

5

5

5

5

5

5

5

5

$\begin{array}{llll}5 & 533 \mathrm{~J} & \end{array}$

GENUS BARLEEIA.

528 B. subtennis, Cpr. Cal.................. 5

529 B. .. var. rimata. Cal......... 5

530 B. haliotiphilla, Cpr. $\quad$. ........... 5

FAMIIY JFFFRIYYSID.E.

GFNES JEFFREYSIA.

531 J. alteri, C’pr. Ćal.

$532 \mathrm{~J}$. bifasciata, C pr.

var. B. "f $\ldots$......

var. C.

var. $\mathrm{D}$.

var. $\mathbf{E}$.

var. F.

var. G.

var. $H$.

var. I.

var. J.
5

5

5

5

5

5

5

5
5
5
5
5
5
5
5
5
5
5

.

\section{FAMILY ASSIMINIIDA:}

\section{EINUS ASSIMINEA.}

534 A. Auberiana, I'Orb. Florida.

5.35 A. concinna, C. B. Ad.

36 A. Californica, J. G. Cooper Cal........ 5 Subgenus Patudinella.

537 P. Newcombiana, Hemphill. Cal....... Io IAMILI TRUNCATELLIDAE. GENUS TKUNCATELLA.

538 T. Californica, I'fr. Cal..

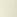

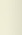

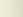

5

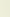

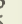

o


GENUS TRUNCATELLA (Continued).

540 T. bilabiata, Pfr. Florida

54 I 'T. pulchella, Pfr. Fla

542 T. " var.

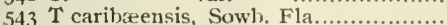

544 T.

\section{FAMII,Y NERITII)}

GFNUS NFRITA.

545 N. peloronta, Lain. I'la.

546 N. " var.

$547 \mathrm{~N}$. " " var.

548 N. " var.

549 N. tessellata, Gmel. Florida............

$55^{\circ} \mathrm{N}$. " var.

55. N. versicolor, Lam.

552 N. " vir.

553 N. scabricostata, Lam. Lower Cal...... GENUS NERITINA.

554 N, reclivata, Say. Florida..

$555 \mathrm{~N}$. virginea, Linn.

$556 \mathrm{~N}$. " var.

557 N. " var.

$558 \mathrm{~N}$. " var.

559 N. viridis, Lam.

FAMILY ADEORBIDA:

GENUS ADEOBRIS.

560 A. supranitidus, var.Orbigneyi, Fisher Florida.

FAMILY CYCIOSTREMATIDAE. GENUS VITRINFLLA.

561 V. subplana. Cpr. California. 562 V. Diegoensis, Dall.

\section{FAMILY LIOTIIDA.}

\section{GFNUS LIOTIA.}

563 L. fenestrata, Cpr. California. 564 L. acuticostata, Cpr.

\section{FAMILY PHASIANELLIDA:}

\section{GENUS PHASIANELLA.}

565 P. umbilicata, D'Orb. Florida 566 P. compta, Gld. California. $567 \mathrm{P}$. $568 \mathrm{P}$.

var. pulloides. California.,

FAMILY TURBINIDA:.

$$
\text { GENUS TURBO. }
$$

569 T. castaneus, Gmel. Florida.

570 T. " var. "

57 I C. fluctuosum. Wood, Lower Cal..... Subgenus Astralium.

572 A. longispina, Lam. Florida.

573 A. Americana. Gmel.

Subgenus Pachypoma.

574 P. inequalis, Mont. California.

\section{GENUS LEPTOTHYRA.}

575 I. Carpenteriana, Pilsbry. California $576 \mathrm{~L}$.

577 L.

$578 \mathrm{I}_{1}$.

579 L, paucilirata, Dall.

$580 \mathrm{~L}$. " var.

581 L. bacula, Cpr. Califorıia.

$582 \mathrm{I}$.

\section{FAMILY TROCHIDAE.}

GENUS CHLOROSTOMA.

$5 \quad 583$ C. Brwneum, Phil. California.

584 C. aureotinctum, Fbs.

585 C. gallina, Fbs, “ “ w....... 15

5586 C. " var. " $\quad$ "......... 15

587 C. . var.tincta. Hemphill.Cal. B. I5

588 C. " var. " " " "

$58 y$ C. " var. " " " " " D. 15

10 590 C. " " var. "funelrale, A. Ad. E. I5

591 C. " var. " " " 5 F. I5

592 C. Montereyi, Keiner. California......

Subgenus Omphalius.

5593 C. viridulum, var. ligulatum, Mke.Cal 10

594 C. fasciatus, Born. Florida............ I5

GINUS NORRISIA.

595 N. uorrisi, Sowb. Califoruia.......... 25

GFINUS MARGARITA.

596 M. pupilla, Gld. Puget Sound.......... 5

597 M. helicina, Fab, Sitka............... IO

$598 \mathrm{M}$, lirulata, var. acuticostata, $\mathrm{Cpr}, \mathrm{Cal}$, Io

$599 \mathrm{MI}$. " var. parcipicta, Cpr. " 10

600 M. " " var, feniculata, Cpr. " "

$601 \mathrm{M}$. " var, sinccincta, Cpr. " 5

602 C., canaliculata, Mart. California ...... 20

603 C. costatum, Mart. " $\quad$..... 15

604 C. auuulatum, Mart. " " $\quad$..... 25

15605 C. supragranosum, Cpr. " " $\quad$..... 20

606 C. tricolor, Gabb. " " ..... 25

607 C. gemmulatur, Cpr. $\quad$ ". $\quad \ldots . .20$

15

25

609 'I. caffea, fiabb.

Floricla.

\section{Subgenus Turcica.}

GENUS ETHALIA.

610 E. supravallata, Cpr. Califoruia....... 6II F. var. invallata, Cpr. Cal .

ro 10

FAMILY HALIOTIDF.

GFNUS HALIOTIS.

6I2 H. rufescens, Swains. California....... 50 6I3 H. Kamtschatkana. Jonas. Washing'n I 0

Io 6I4 H. corrugata, Gray. Califormia......... 50

6I5 H. assimilis, Dall. " "....... I O

6 I6 H. splendens, Rve, $\quad$. $\quad$......... 50

6 I7 II. cracherodi, Leach, " ....... 50

FAMII, FISSURELIIDE.

IO

IO

25

25

25

25623 L. cremulata, Shy. California

624 . fasciata, Pfr. Floricla..................

5

5

5

5 20

Io $629 \mathrm{~F}$. calliomarginata, Cpr. California...

$5.629 \mathrm{~F}$. bimaculata, Dall.

5630 F. limatula, Rve. Florida.

\section{GENLS GLYPIIS.}

625 G. aspera, Esch. Califorıia............ 20

626 G. pica. Sby. Lower "“ .......... 25

627 G, nurina, Cpr. $\quad$ “ ........... 25

\section{GENUS FISSLRELLID.E.}


FAMILT ACMAIDE.

GENUS ACMEA.

631 A. puuctulata, Gmel. Florida..........\$ 10

632 A. mitra, Esch, California.

$633 \mathrm{~A}$, instabilis. Gld. Subgenus Collisella.

634 A, pelta. var. Nacelloides, Dall, Cal...

635 A. " var.

$636 \mathrm{~A}$, “ var.

$638 \mathrm{~A}$. " var.

639 A. " var.

$640 \mathrm{~A}$. " var.

64 I A. patina, Esch

642 A. " var.

643 A. " var.

644 A. scabra, Rve.

645 A. " var.

$646 \mathrm{~A}$. " "var.

647 A. " " var. Morc hii. Dall. A. “ ...

649 A. asmi, Midd. California.

650 A persona, Esch.

65 I A. " var.

652 A. " var.

653 A. " var.

655 A, spectrum, Nutt.

$656 \mathrm{~A}$, " var.

657 A. " v var.

$65^{8} \mathrm{~A}$. " " var.

659 A. “ var.

660 A. depicta, Hds.

66I A. paleacea, Cpr.

662 A. triangularis, Cpr.

GENUS LOTTIA.

663 L. gigantea, Gray. California.
$637 \mathrm{~A}$. " var.

648 A. " var. " Dall. B. " ...

654 A. " var.

Subgenus Maugerella.

677 M. conspicna, Cpr. California... .....\$0 20

$678 \mathrm{M}$. " var. A. acrior, Cpr. Cal 30

$679 \mathrm{M}$.

var. B. "Cpr." 30

GENTS ISCHNOCHITON.

680 I. Cooperi, Cpr. California.............. 20

68I I, regularis, Cpr. " $\quad$.............. 25

682 I. papillosus, C. B. Ad. Florida........ 20

683 I. funiculata, Cpr. " $\quad$..... 20

Subgenus Stenoplax.

684 S. lamiciformis, Sowb. Florida........ 25

Subgenus Ischnoplax.

685 I. pectinulatus, Cpr. California........ I5

686 I. " var. " ......... 15

Subgenus Lepidopleurus.

687 L. Mertensi, Cpr. California.......... 25

GENUS CALLISTOCHITON.

688 C. fimbriatus, Cpr. California........ 20

689 C. palinulatus, Cpr. " " $\quad$......... 20

690 C. decoratus, Cpr. “ $\quad$ "....... 15

GENUS PALLOCHITON.

691 P. lanuginosus, Cpr. Lower California 25

ACANTHOIDEA.

GENUS ANCANTHOPLEURA.

692 A. piceus, Gmel. Florida.

GENUS NUTTALLIANA.

693 N. scabra, Rve. California............ 15

694 N. " var. " ............ I5

\section{CHITONES IRREGULARES,}

I O P A L OI DEA.

GENUS MOPALIA.

695 M. ciliata, Sowb. $=$ muscosa. Gld. Cal. 25

696 M. “ var. Califormia............... 25

697 M. , var. ". $\quad$................. 25

698 M. “ var. "

699 M. lignosa, Gld. “ $\quad$................... 25

700 M. " var. acuta, Cpr. California 25

701 M. " var. " 25

702 M. Hindsii, Gray. $\quad$ “ 25

703 M. “ var. " " 25

704 M.Wossnessenski, Midd.=Kennerleyi, Cpr. California................ 25

705 M. Wossnessenski, var. California..... 25 Subgenus Placiphorella.

706 P. velata, Cpr. California............... 25

GENUS KATHERINA.

IO

10

10

10

Colored varieties.

GENUS TONICEILA.

669 T. lineata, Wood. California.

GENUS CHATOPIEURA.

670 C. Hartwegii, Cpr. Califormia...........

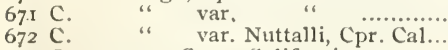

673 C. gemmea, Cpr. California............

674 C. " var " $\quad \ldots \ldots \ldots \ldots . . .$.

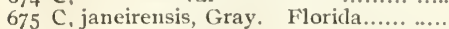

676 C. apiculatus, Say.

IO

IO

IO IO

IO

Io

20712 A. puructatus. D'Orb. Galifornia. 


\section{GENUS TORNATINA.}

713 T. culcitella, Gld, California.

7 I4 T. inculta, Gld.

715 T. " var.

716 T. harpa? Dall.

$717 \mathrm{~T}$. carinata, Cpr.

718 T. candei, D'Orb. Florida

7 I9 T. canaliculata, Say. “ .....................

$720 \mathrm{~T}$. infrequens, Cpr. Lower California Subgenus Rictaxis.

72I R. punctocælata, Cpr. California......

FAMILY CYLICHNIDAE.

GENUS AMPHISPHYRA.

722 A. subquadrata, Cpr. California..

FAMILY BULLIDA:.

GENUS BULLA.

723 B. nebulosa, Gld. California.

724 B.

725 B. striata, Brug. Florida.

$726 \mathrm{~B}$. occidentalis? Adums. Florida

727 B, succinea, Conr.

Florida.. ...

GENUS HAMINEA.

728 H. vesicula, Gld. California..

729 H. virescens, Sby.

730 H. Guildingi, Sowb.

GFNUS CYLINDROBULLA.

73I C. Beaui, Fisher, Florida

FAMILY APLYSIIDF.

GENUS APLYSI.A.

732 A. protea, Rang. Florida.

733 A. Californica, Cooper. California....

\section{FAMILY AURICULIDIE.}

GFNUS CARYCHIUM.

734 C. exiguum, Say. Minnesota.

$735 \mathrm{C}$. $\quad$ sp, Washington, Org. GENUS AURICULA.

Subgenus Auriculastrum.

736 A. pellucens, Mke. Florida

$$
\text { GENUS TRALIA. }
$$

Subgenus Alexia.

737 P. myosotis, var.setifer J.G.Cooper.Cal GENUS PEDIPES.

738 P. uaticoides, Stearns, Florida.

739 P. elongatus, Dall. $\quad$ *........

740 P. unisulcatus, J. G. Cooper, California

\section{GFNUS MELAMPUS.}

74 I M. coffeus, Linn. Florida

$742 \mathrm{M}$.

$743 \mathrm{M}$.

var.

var.

744 M. flavus, Gmel.

745 M. lineatus, Say.

$746 \mathrm{M}$. olivaceus, Cpr, California

$747 \mathrm{M}$.

$748 \mathrm{Mr}$.

$$
\text { " var. " }
$$

Subgenus Detracia.

749 D. bulloides, Mont. Florido

Subgenus Sayella.

750 S. Hemphilli, Dall. Florida
757 D, hexagonum, Sby, California.

758 D autillarium, D'Orb. Florida....... 15

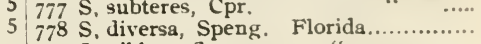
779 S. gibbus, Speng.
FAMILY SOLENIDA: GFNUS SOLFN.

$772 \mathrm{~S}$, Americauus, Gld, Florida........... 20

774 S. .. var, rosaceus, Cpr. Cal.. I5 GFNUS SILIQUA.

Dixon. Oregon...
cifints SOL RCURTUS.
773 S. sicareus, Gld. California.............

\section{FAMILY SAXICAVIDAE.} GENUS SAXICAVA.

780 S. arctica, Linn. Californis 
GENUS PANOPEA,

78I P. generosa, Gld. Puget Sound......

FAMIILY MYACIDAE. GENUS MYA.

$782 \mathrm{MI}$, arenaria, Linn, California........... 783 M. truncata, Linn. Puget Sound..... GENUS PLATYODON.

TSt F, cancellatus, Conr. California....... FAMIIY CORBULIDA. GENUS CORBULA.

$7 \mathrm{~S}_{5}$ C. Iuteola, Cpr. California.. $786 \mathrm{C}$. " var. " 787 C. contracta, Say, Florida. $788 \mathrm{C}$. $\quad \mathrm{sp}$.

$789 \mathrm{C} . \quad \mathrm{sp}$.

GENUS NEARA.

790 N. costellata, Desh. Florida GEXUS CRYPTOMYA.

791 C, Californica, Conr, California.....

FAMILY ANATINIDE. GENUS CLIDIOPHORA,

792 C. punctata, Cpr. California. GENUS ASTHENOTHFRUS.

793 A. Hemphilli, Dall. Florida. GENUS THRACIA.

794 'T. curta, Conr. California. GENUS PERIPLOMA.

795 P. argentaria, Conr, California GENUS LYONSIA.

796 L. Californica, Conr. California........ 797 L. hyalina, Conr. Florida

Subgenus Entodesma.

798 E. inflata, Cpr. California.

799 E. saxicola, Baird,

GENUS MYTILIIMERIA,

800 M. Nuttalli, Conr. California

\section{FAMILY MACTRIDA: GENUS MACTRA.}

8ot M. solidissima, Dill. Florida.

802 M. lateralis, Say.

\section{Subgenus Hernimactra.}

$80_{3}$ M. fragilis, Chem, Florida

804 M. nasuta, Conr. California............

805 M. planulata, Conr.

806 M. falcata, Gld.

807 M: Calitornica, Conr. California ........ GENUS SCHIZOTHARUS.

808 S. Nuttalli, Conr. California.

\section{FAMILY SEMILII.F!. GENUS SEMIIE.}

Sog S. decisa, Cont. California...

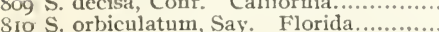

8I I S, obliqua, Wood,

\$12 S. reticulata, Gmel.

813 \$. radiata, Say.

8 I 4 S. cancellata, Y'Orb.

(n..........

cancellata J'Orb. "6 c.........

8 I5 S. pulchra, Sby. California.

816 S. rupium, Shy.
I 00

GENUS SYNDOSMYA.

817 S. aqualis, Say. Florida.............. I6

GENIS LUTRICOLA.

818 L. alta, Conr, Califormia............... 25

20

8ı9 C.. subdiaphana, Cpr, California..... GENIS CUMINGIA,

820 C. Californica, Cpr. California........ 15

821 C. tellinoides, Conr. Florida.......... 20

FAMILY TELLINIDE,

GFNUS GARI.

IO

IO

IO

IO

823 S. Nuttalli, Conr. California...

GFNUS TELLINA.

20824 \%. Bodegensis, Hds. California.

S25 T alternata Say. Florida........

826 T. Braziliaua, Lam. " $\ldots \ldots \ldots \ldots \ldots . .15$

827 \%. " " var. "

828 T'. punicea, Born, Lower California..... 25

829 I: levigata, Lin1. Florida............. Io

s3o $T$. decora, Say. " $\quad$ "........... 25

$258_{31}$ T. Martinicensis, D'Orb. Florida...... 25

832 T, Sonleytiana, Recl, " $\quad$..... 25

25 Subgenus Angulus.

833 'r. polita, Say. Florida.................. Io

25: 834 T. variegata, Cpr. Califorıia.......... 15

835 T. obtusus, Cpr. Puget Sound......... I5

836 \%. modestus, Cpr. California........... 15

837 '. Gouldi, Cir. "

IO GENLS STRIGILI,IA.

Io 838 S. flexuosus,Say. Florida...

CiF.NUS MACOMA.

25839 M. fausta, Desh. Florida.

840 M. secta, Conr. California............. 25

84 I M. constricta, Brug. Florida.......... 25

25842 M. proxima, Gray. " $\quad$.......... 25

843 M. nasuta, Conr. California.......... 25

844 M. inquinata, Cpr. " ......... 20

845 M. inconspicua, Br. \& Sby. Cal.... ... Io 25 $846 \mathrm{MI}$.

var. red.

GENUS TELLIDORA.

847 'T. lunulata. Holmes. Florida........... 25

FAMILY DONACIDA:. GENUS DONAX.

$40 \quad 848$ D. variabilis, Say, Florida

85 I D. " var, va va.............

50852 D. Californicus, Conr. " $\quad$............ 5

853 D. " " var, " "

854 D. " " var. "

855 D. " var. "

856 I). punctostriatus, Hanley. Lower Cal Io

857 D. flexuosus, Gld. California.......... Io Subgrnus Heterodonax.

858 I1. bimaculatus, D'Orb. California... 5

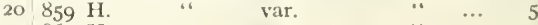


FAMILY PITRICOLID $A$ :.

GENUS PETRICOIA.

86I P. cardatoides, Conr. California........ 862 P. " var. " ....... $863 \mathrm{P}$. divaricata, D'Orb. Florida............ 864 P. tenuis? Ad. Lower Califormia...... GFNUS RUPFI,LARIA

865 R. lamellifera, Conr. California GENUS SAXIDOMUS,

866 S. Nuttalli, Conr. California.

867 S. " var. aratus, Gld. California FAMIIY VENERIDA: GENUS VENUS.

868 V. mercenaria, Linı. large. Florida.... GENUS CRYPTOGRAMMA.

869 C. flexuosus, I,inn. Florida. Subgenus Chione.

870 C. gnidia, Brod. \& Sowb. Lower Cal... 87 I C. succincta, Val. California.

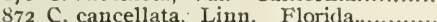

873 C. simillima, Sby. California.

874 C. " var. undatella, Sby. I. Cal

875 C. fluctifraga, Sby. California............ Subgenus Psephis.

S76 P. tantilla, Gld. Califoruia GFNUS CYTHFRIA.

877 C. affinis, Gmel. Florida.

878 C. " var.

Subgenus Transenella.

879 T. couradiana, Dall. Florida.

s8o 'T. Cubaniana, D'Orb. Florida. Subgenus Callista.

88I C. gigantea, Chem. Floricla.

$88_{2}$ C. maculata, Chem.

$88_{3}$ C. incerta, Desh.

8S.4 C. chioue, Mke. Lower Cal. Subgenus Tivela.

885 T. radiata, Sby. Lower California....

$886 \mathrm{~T}$. crassatelloides, Conr.

Subgenus, Amiantis.

887 A. callosa, Conr. California... Subgenus Gouldia.

888 G. cerina, Ad. Florida. Subgenus Circe.

889 C.? marginata, C $\mathrm{pr}$. California GFNUS DOSINA.

890 D. ponderosa, Gray. Lower California.

89 I D. elegans, Cour. Florida,.............. GENUS TAPES,

892 T. tenerrima, Cpr. Puget Sound........ 893 T. laciniata, Cpr. California.

894 T. staminea, Conr.

$395 \mathrm{~T}$

896 T.

$897 \mathrm{~T}$. var. diversa, var. orbella, var.ruderata, “

$898 \mathrm{~T}$. var. Petitii,

S99 T. grata, Say. Lower "

900 T. " var. " "

$90 \mathrm{I}$ T. " var. " "

902 T. " var. 6"

903 T. " var. " " "

904 T. " var. "

$905 \mathrm{~T}$. " distorted, " "

I regard T grata, as only a Southern form of T sta... IS
FAMILY CYRENIDA:

GENUS CYRTNA,

I5 906 C. Caroliuensis, Conr. Florida.

I5 907 C. Floridana, Jam.

FAMILY CARDIIDA:

GINUS CARDIUM.

25 go8 C. magnum, Born, Florida............. 25

gng C. isocardia, Linn. “ ........... 25

259 Io C. muricatum, Linn. " * ......... 25

25 gII C. corbis, Mont. California............. 25 912 C. quadragenrsium, Conr. California .. 50

Subgenus Liocardium.

50 913 I. elatum, Sby. California........... I ou

9I4 I. serratım, Linn. Florida............ 50

${ }_{5} 9$ I 5 I. Mortonii, Conr. Florida............. Io

916 I. " var. substriatum, Conr.Cal 10

40

20

20

20

20

o

20

20

922 L. Jamaicensis, Speng. Florida ........

923 I antillarium, Rave.

924 L. strigilla, Stimpson.

FAMILV CIIAMIIA:

GENUS CIIAMA.

917 C. arcinella, Linn. Florida ............ 25

9I8 C. macrophylla, Chem. " ............ I5

9 I9 C. pellucida, Sby. California......... I5

920 C. " var. spinosa, Shy. Cal... I5

921 C. " var. exogyra, Conr. "... IO

FAMII, IUCINIDA.

GFNUS LUCINA.

20

25

925 I. lintea, Conr.

926 L. squamosa, Lam.

927 I. crenulati, Lam.

928 1. pecten, I.an.

929 1. Pennsylvanica, Linn. ….... 25

930 L. Californica, Conr. California....... 20

931 1. Nuttalli, Conr.

Subgenus Codakia.

932 1. tigerina, Limn, Florida ............ 25

Subgenus Loripes.

25933 I.. Floridana, Conr. Florida

FAMII,Y DIPLODONTIID.F.

\section{(iFXE'S DIPLODONTA.}

Io $93+1$. orbella, Gle. Cialiforuia..

935 1). candeana, D'Orh. Florida.

Subgenus Felania.

936 I. sericata, Rve. Lower California
FAMIIS FRYCINIDA.

GFNeS MOXTACUTA.

Subgenus Tellimya.

937 T. tumida, Cpr. California.

EFXI'S LASSFA.

938 L. rubra, Mont. Califormia............

GFXUS KFI, I,IA.

939 K. Laperousii, Desh. California...... 20

940 K. suborbicularis, Mont. $\quad$ " ...... 15

FAMILY SOI,EMYIDA: GFXY'S SOLFMYA.

94 I S. occidentalis, Desh, Florida

942 S, voloulus, Cpr. Lower Cal....... 
FAMILY CRASSATELLIDE. GENUS EURIPHYLA.

943 E. lunulta, Conr. . 15

FAMILY ASTARTIDA.

GENUS PARASTARTE.

944 P. triquetra, Conr. Florida

FAMILY CARDITIDE. GENUS CARDI'TA.

945 C. Floridana, Conr. Florida. GENUS MILNERIA. 946 M. minima, Dall. California.

Subgenus Thecalia.

947 T. coucamerata.? Chem, California... GENUS CARDITAMERIA.

948 C. subquadrata, Cpr. Califoruia......... FAMIILY NUCULIDE. GENUS NUCULA.

949 N. crenulata, A. Ad. Florida $950 \mathrm{~N}$. exigua, Sby. Lower Califoruia....

$$
\text { GENUS LEDA. }
$$

95I L. acuta, Conrad. Califoruia............

\section{FAMILY ARCIDE.} GENUS ARCA.

952 A. multicostata, Sby. Lower Cal ......

953 A. ponderosa, Say. Florida.............

954 A. Reeviana, D'Orb. Lower Cal ......

955 A. solida, Sby.

956 A. Lesteri, Phil, Florida.

957 A. transversa, Say,

$95^{8}$ A. Deshaysii, Hanley.

Subgenus Barbatia.

959 B. gradata, Sby. Fla., Cal, and L. C.. GENUS PECTUNCULUS.

960 P. pectiuatus, Lam. Florda. 96I P. intermedia, Brod. Califoruia...........

\section{FAMILY MYTILIDA. GENUS AYTILUS.}

962 M. edulis, Linn. California \& Wash 963 M. " var. Puget Sound............. 964 M. Californicus, Conr. California...... 965 M. bifurcatus, Conr. " ....... 966 M. exustus, Linn. Florida .............. I5 GENUS MODIOLA.

967 M. capax. Cpr. California.............. 968 M. " var. Lower "

969 M. sulcatus, Lam. Florida................

970 M. plicatula, Lam.

971 M. tulipa, Linn.

972 M. fusca, Gmel.

(n)

973 M. recta, Conr.

974 M. " var. flabellatus, Puget Sound

975 M. papyracea, Conr. Florida............

Subgenus Adula.

976 A. falcata, Gld. California.

977 A. stylina, Cpr.

GENUS LITHODOMUS.

978 L, attenuatus, Desh. Lower Califoruia 979 L. appendiculatus, Gmel. Florida...... GENUS MODIOLARIA.

980 M. lateralis, Say. Florida... GENUS SEPTIFER.

981 S. bifurcatus, Rve. California..

FAMILY AVICULIDA: GENUS AVICULA.

982 A. atlantica, Lam. Florida.

5

10
GENUS MELFAGRINA.

983 M. radiata, Lam. Florida...............\$ 25

GENUS PFRNA.

984 P. ephippium, Lam. Florida.......... I5

985 P. chemuitzian, D'Orb. Lower Cal... I5 FAIIIYY PINNIDE.

GENUS PINNA.

986 P. muricata. Linn. Florida............ 35

Io 987 P. carnea, Gmel. " . ............ 50

GENUS BRYOPHILA.

20 988 B. setosus, Cpr. Califormia............. 40

FAMILY SPONDYLIDA.

GENUS PLICATULA.

989 P. ramosus, Liun. Florida.

FAMILY LIMID.E. GENUS LIMA.

990 L. inflata, Lam. Florida ................ 25

991 L. orientalis, Califoruia

I:AMILY PECTINIDA. GENUS PECTEN.

992 P. subuodosus, Sowb. Lower California 993 P. nucleus, Born. Florida................ $99+$ P. " var. aquisulcatus, Cpr.Cal. 995 P. " var. " Cpr. "

997 P. exasperatus, Sowb.

$998 \mathrm{P}$. " var.

999 P. ornatus, Lam.

1000 P. latiauritus, Conr. California......... IOOI P. " var.

1002 P. monotimeris, Cour.

1003 P. " var.

atus, Say. Fla...

Fla... 25

20

20

20

25

50

50

20

20

20

20

20

GFNUS HINNITES.

15
25

I005 H. giganteus, Gray, California.

50

FAMILY ANOMIIDAE.

GEXU'S ANOMIA,

I006 A. ephippium, Linn. Florida.......... I5

25

15

15
I016 G, antillarium, var, pyrimidata,
Stimp. Florida ................... 50

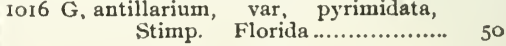

Ioo8 P. macroschisma, Desh, Californa... 25

FAMILY OSTREID.F. GFNUS OSTRFA.

ı009 O. lurida, Cpr. California............. Io IOIO O. " var. expansa, Cpr. Cal.... 10 IOI I O. amara, Cpr. Lower California..... 20 1012 O, conchaphilla, Cpr. " $\quad$.... 15 IOI3 O. palmula, Cpr. " "

\section{CLASS BRANCHIPODA.} FAMILY DISCINIDA.

GENUS DISCINA.

1014 D. cumingii, Brod. Lower California 50

FAMILY LINGULIDA.

\section{GENUS GLOTIDE.}

1015 G. albida, Hds. California............ 50

\section{ORDER BRYOZOA. \\ FAMILY DISCOPORIDA. GENUS DEFRACINA.}




\section{LAND SHELLS.}

\section{PULMONA'IA.}

\author{
I:AMILT TESTACELI,IDA: \\ GENUS GLANDINA.
}

IoIS G. truncata, Gnel. Floricla..........\$\$ 25 $1019 \mathrm{G}$.

\section{FAMHLY SELENITII)A: \\ (IENUS SELENITHS. \\ A. Smooth forms.}

1020 S. concava, Say. Ohio, Ky., Tenn.\&N.C

1021 S. " " var. small. ()hio

1022 \$. " var. Henphilli, WV.G.Minı

Waslinigton.

1023 S. " " var. Keepi, IIenipliill.Cal

1024 S. " " var, telluis,

$1025 \mathrm{~S}$.

$1026 \$$

var.occirlentalis, "

" var.Vanconverensis, sulall

Oregon.....

1027 S. “ var. Vanconverensis, Lea.

\section{large. Oregon...................}

B. Forms with reticnlated sculpting.

Io2S S. concava, var. hylırida, Henpliill, Or.

1029 S. " " var. sportella, large. Wash

I030 S. " " var. " typical,

103 I S. " " var. " smaller, "

1032 S. " " var. " v. small "

1033 S. " v var. Voyana, Newc.

I034 S,

I035 \$.

var. ". small.

var. transfuga, Hemphill.

I036 \$. concava, var, simplicilabris, Ancwy

Califorıia........................

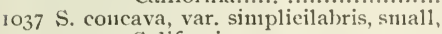

Califorıia.

$103 \mathrm{~S}$ S. concava, var. crelata, Mazyck. Cal.

I039 S. " var. Duranti, Newc.

I040 \$. " var. Catalinensis, Hen1p-

hill. California

FAMIIT IIMACII).E. GHNUS ZONITES.

Section Mesomphix. Depresserl.

1041 Z. subplanus, Binn. N. Carolina...... 1042 Z. inornatus, Say. Ky.

Subelevated.

1043 Z, Lrevigatıs, I'fr. N, Caroliı......... 1044 Z. Rugeli; W. G. Biı11. 'Ten11 .......... jo 45 Z. copnotes, IV. G. Binis.

Elevated.

I046 7. ligerns. Say. Olıio.

$1047 \%$ " " var, tlenissus, Binu.......... Ala

1048 Z. " var, acerus, Lewis, Ga...

I am strongly impresed that all the above

Zonites are but varieties of one sprecies.

1049 Z, Elliotti, Rerlfield. N, Carolina...... I050 $\%$.

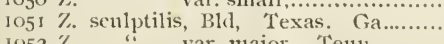

$1052 \%$. "Tar. major. Ten11 ...........

\section{Section Hyalina.}

s053 Z., nitidus, Mull. Cal., Minsı., Etalı.. 1054

Z. var, arboreus, Say, C’al.,

Ttali..........................

GWXUS ZoNITF\% (Continued.)

I055 7. 11itidus, var.cronk heitei, Newc. Cal. son o5 $1056 \mathrm{Z}$. " var. otonis, Florida..... 5

1057 \%. petrophilus, Bli. N. Carolina........

Io5s 7. Wheatleyi, Bhl.

$1059 \%$.

$1060 \%$ capsella, (rld.

1061 \%. W'nitneyi, Newc. California, Utah. 25

$1062 \%$ indentatus, Say. Florida.......... I5

$1063 \%$, viriclulus, Mke, WVashingfon ......

I064 \%. minusculus, Morse. Florida, La..

I065 \%. 111ilum ? Morse. California..........

I066 \%. pygninea, I)rap.

\section{Section Conulus.}

1067 7. fulvus, I) rap. Cal., L'talı.......... 5

I068 \%. " var. Gundlachi, Pfr. Fla.. I0

1069 \%. cliersinellus, Dall. Califorıia..... 25

Scction Gastrodonta.

15

I093 P. Andersonii, J. G. Cooper. Wash...

5 log. P. Henplsill, W. G. Binn.

In95 P. ․ var, cieruleum, Cockrell.

1070 Z. gularis, Say. N. Caroliua, Tenu.. 1071 Z. interu1us, Say. Georgia, N. C...... 1072 \%. nacilientus, Slutt. Tennessee... 1073 Z, suppressus, Say. Florirla............ 1074 Z. Anclrewsi, W. C. Binn. Tenı.... $1075 \%$ significans, Bld. Nortlı Carolina.. Section Pristiloma.

$1076 \%$ I, Iansingi, Bld. Waslıington ........ GFNUS VITRIZONITES.

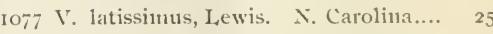
GENUS VITKINA.

Io $\$$ S V. Pfeifferi, Newe. Cal., Ëtah........ 20 GENUS LIMAX.

1079 I. nuaximus, I.inu. N. Jersey......... 25

Joso J. Hewstonii, J. G. Cooper. Cal...... 20

IOS1 I. Henphilli, VV. G. Binis. Cal..... 20

IoS2 L. canipestris, Binn. Washington... 15

$\operatorname{loS}_{3}$ L. .. var. Montanus. Ingersoll. [tah $\ldots \ldots \ldots \ldots \ldots \ldots \ldots$. 15

Ios.t I. canpestris, var. byperborens, Viest. Oregon...................

FAMII, TIRENNOPHORIJ.E. GFXIS THBHXXOPHORE'S.

ros 5 T. ca:olinensis, Bose. Olio............

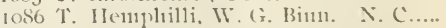

\section{lFAMII, ARIONIID.E.}

GiNis's ARIOI,IAX.

ros7 A. Colnumlianus, Cild, Oregon, Čal... 25 uoss A. ". var. nuculatus, Cockrell, C'slifornia................. rotig A. Columbianus, var. Californicus, J. (i. Cooper. California...... sogn A. Colnumbianns, var. straminea. I091 A. niger, J. G. Cooper. California.. 1092 A. Heniplitli. WV. G. Binu. Cal..... (IFNIS PROPHTSAOX. 
GENUS PROPHYSAON. (Conntinued). rog6 P. Hemphilli, var, fasciatum, Cockrell, Washington.............. GENUS HEMPHILLIA.

I097 H. glandulosa, Bld. \& Binn. Oregon. Io9s $\mathrm{H}$. " large. Idaho............ GENUS BINNEYA.

I099 B. notabilis, J. G. Cooper. Cal.(shell) FAMILY HEIICIDE.

\section{GENUS HELIX.}

\section{Section Glyptostoma.}

I Ioo G. Newberryanuщ, W. G. Binı. Cal. I place this specie nere on account of the shell being that of a Zonites, while the animal is said to be true Helix.

\section{Section Microphysa.}

I IOI H. Ingersolli, Bld. Utah, Oregon...... I $102 \mathrm{H}$. votex. Pfr. Florida.

\section{Section Gonostoma.}

I 103 H. Yatesii, J. G. Cooper. California I 104 H. " var. small.

\section{Section Helicodiscus.}

I 105 H. lineata, Say. Minnesota........... I106 H. " var. fimbriatus, Wetherby.

$$
\text { Georgia..... }
$$

I $107 \mathrm{H}$.

" var. salmonensis, Hemphill.

$$
\begin{aligned}
& \text { Idaho. } \\
& \text { Section Polygyrelta, }
\end{aligned}
$$

I $108 \mathrm{H}$. polygyrella, Bld. Idaho.

I $109 \mathrm{H}$.

$$
\text { var. }
$$

\section{Section Polygyra.}

GROUP OF H. CFREOLUS MUHLF, $1110 \mathrm{H}$. cereolus, Muhlf. Florida.

IIII $\mathrm{H}$. " " var. ".......... III2 H. " " var.

$1113 \mathrm{H}$. $1114 \mathrm{H}$. I I $15 \mathrm{H}$. $1116 \mathrm{H}$. II $17 \mathrm{H}$. III $8 \mathrm{H}$. II $19 \mathrm{H}$. $1120 \mathrm{H}$. I 2 I H. var. "var. septemvolva, Say. Fla. "var. " " medium... " var. " small ...... "var. Carpenteriana, Bld...... " var. " small..... " var. volvoxis, Parr, " .... " var. " small ........... " var. microdonta, Pfr........... GROUP OF H. TEXASIANA.

II 22 H. var. triodontoides, Bld. Texas.... 1123 H. var. Dorfeuilliana, Lea. " .... II 24 H. var. Mooreana, W. G. Binn......... GROUP OF H. AVARA.

1125 H. avara, Say. Florida I $26 \mathrm{H}$ auriformis, Bld Lousians........... I 27 H. pustula, Fer. Florida.................. GROUP OF H. AURICULATA.

I 28 H. var. uvulifera, Shutt. Florirla..... I129 H. var " smąll.

\section{Section Patula.}

GROUP OF H. PERSPFCTIVUS,

I $30 \mathrm{H}$. perspectivus, Say. Ten11............. I I 3 I H. I I $32 \mathrm{HI}$. I I33 II. var. elevated. Teun.... var. Bryanti, I Iarper. ".. var. striatella, Athy,... Minnesota

II34 H. "var.Cronkheitei, Newc. Utah I 35 H. astericus, Morse. Washington ... II36 H. * var. conspectıs, Bld,Cal,

\section{GROUP OF H SOLITARIA.}

I $137 \mathrm{H}$. solitaria, Say. large. Idaho........ I5

25 I 38 H. " var. small, elevated...... IO

$1139 \mathrm{H}$, “ var. depressed............... I0

25 I1 $40 \mathrm{H}$. "“ var. light colored......... IO

50 IO4I H. " " var. " depressed....... I0

II42 H. " " var. bandless............. IO

I 43 H. alteruata, Say. Min11, Ohio, Texas. Io

GROUP OF H. STRIGOSA.

A. sliell trausversely ribbed.

I 44 H. strigosa, var. Idahoensis, Newc. Idaho I $145 \mathrm{H}$. ". var. " small, depressed, Idaho...............

I $146 \mathrm{H}$, “ var, Idahoensis, small, elevated. Idaho.

II 47 H. " var. Idahoensis, depressed,

15 I $14 \mathrm{~S} \mathrm{H}$.

I5 II $49 \mathrm{H}$.

I $50 \mathrm{H}$.

I5 II5I H. 5 I $152 \mathrm{H}$. 20

IO

I 53 H. strigosa, var.multicostata, large. I 154 H. "var. " smaller. Utah I $55 \mathrm{H}$. " var. " " depressed" II56 H. * var. " " small. 20 II57 H. " v var. " smaller. " 20 I158 H. " " var. " smallest. "

II 59 H. strigosa, var. multicostata, Heinp-
IO IO

IO IO

IO

IO

IO

IO

IO

Io

IO

\section{I $66 \mathrm{H}$}

15

I5 II67 H
I5 $1169 \mathrm{H}$. I $70 \mathrm{H}$.

15 II I H.

20 II72 H.

I $73 \mathrm{H}$.

I $74 \mathrm{H}$. I $75 \mathrm{HI}$.

Io

Io I176 H. strigosa, var. Binneyi, Hemphill.

$5 \quad 1177 \mathrm{HI}$. " var. " smaller "
" var. Idahoensis, large. Idaho

" var. Newcombi, Hemphill, Utah. hill. Utah.....................

var, multicostata, elevated Utah.

" var. multıcostata, delicate, depressed. Utah.

" var. multicostata, elevated, siugle band. Utah var. multicostata, faintly banded. Utah................

" var. multicostata, clonded, \&c. Utah.

"var. nutticostata, dentate, Utah.

" multicostata, small; ele'ted Utali............................. multicostata, depressed,

X. Ribbing variable. sa, var. Gouldi, Hemphill,

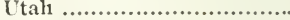

var. Gouldi, Utah.... var. Gouldi, dentate, Utah. "var. " conical " " var. " ilepressed ". "var. " elevated " "var. " very small " X. Bandless forms, Utali... var. Binneyi, Hemphill. Utah ......................... "var. Binneyi, Henphill. Tralı............................ 
GROUP OF H. STRIGOSA. (Continned). I 79 H. strigosa, var. Binneyi, depressed. I 80 H. " var.............................. Binneyi, elevaterl. II81 H. " var. Binneyi, depressed Utah.............................

I182 H " var. Binneyi, dentate.

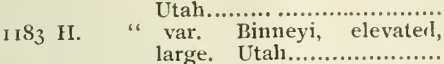

IIS4 H. " var. Binneyi, depressed, large. Utah.

X. Banded forms, ribbing variable. I S5 $_{5}$ H. strigosa, var. albofasciata, Hemphill. Utah.....................

I $86 \mathrm{H} . \quad$ " var. albofasciata, large
elevated. Utah.................
I $87 \mathrm{H} . \quad$ var. albofasciata, smaller

I $86 \mathrm{H} . \quad$ "var. albofasciata, large
elevated. Utah.................
I187 H. "var. albofasciata, smaller depressed. Utah..............

I I8S H. " var. albofasciata, elevated Utah..........................

I 89 H. " var. albofasciata, smaller rtah...........................

I 190 H. " var. albofosciata, lentate Utal...........................

II9I H. " var. albofasciata, very small. Utalı...................

II92 H. " var. castaneus, Hemphill. Utah.

II93 H. "var. castaneus, smooth. Ore.
X. Shell keeled, ribbing irregular.

I 94 H. strigosa, var. Wahsatehensis, Utalı I $195 \mathrm{H}$ var.

tall,

narrow. Utalı.

I $196 \mathrm{H}$. “ var. Wahsatchensis, small, Utah.

I 97 H. “ var. intersum, Hemphill, Idaho.

II98 H. " var. intersum, small.Iilalio. B. Shell smooth or with rough strice. Keeled.

I I99 H. strigosa, var. Utahensis, Hempliill, Utah

I $200 \mathrm{H}$. “ var: Utahensis, smal], Utah X. Subcarinated forms.

I 20 I H. strigosa, var. subcarinata, Iclalıo.. I 202 H. " var. picta, Hemphill, " I 203 H. " " var. bicolor,

1204 H. " " var. " depressel, "

$1205 \mathrm{H}$. " " var. lactea,

I206 H. " var. parma, Washington, X. Whorls convex, stria rollgh. 1207 H. strigosa, var. rugosa, Hempliill... I208 H. " var. "“ depresserl... $1209 \mathrm{H}$. I 2 IO $\mathrm{H}$. I 2 I I $H$. I 2 I $2 \mathrm{H}$. $1213 \mathrm{H}$. $1214 \mathrm{H}$. I2I $5 \mathrm{H}$ $12 \mathrm{I} 6 \mathrm{H}$. I 2 I $7 \mathrm{H}$. var. " smaller...... var. " smallest.... var. Cooperi, WV. G. Binı. var. var. var. var, fragilis, Hemphill.. var.. depressed... var. " small.

I2IS H strigosa, var Buttonii, Hemphili,
GROUP OF H. STRIGOSA. (Continued).

I 2 I9 H. strigosa, var. Buttonii, smaller. Utah I220 H. " var. " " depressed. "

122 I H. " var. "

$1222 \mathrm{H}$. " var. " smaller.

1223 H. " var.

1224 H. " var. " "

1225 H. " var. " very small.,"

Typical forms of $\mathrm{H}$. strigosa.

1226 H. strigosa, Gld, [tah.................

1227 II. ." "

$1228 \mathrm{H}$.

122911.

$1230 \mathrm{H}$.

I23I H.

1232 II.

$1233 \mathrm{II}$.

I234 1 I.

$1235 \mathrm{H}$.

larger.

variable.

“ large.

var. jugalis, Ilemphill. [tah.

var. " medium.

var. " small.

Bandless forms,

I236 II. strigosa, var. cormeus, Hemphill. I237 H. “ var. “ I238 II. " " var. " "lepressed... 1239 H. " var. " variable... 124011 .

I24I II.

124211 .

I $2.43 \mathrm{II}$.

$1244 \mathrm{H}$.

I 245 II.

C. Shell with revolving ribs Subangled or keeled.

I246 HI. strigosa, var. lybbrida, I Iemphill.

I247 H. "“ var. " " elevated.

I24S II. “ var. “

I249 II. “ v var. Hemphilli, Newc....

I250 H. “ var. " “ smaller...

I25I II. “ $\quad$ var.Oquirrliensis, IIempliill.

1252 II.

$1253 \mathrm{HI}$.

I254 H.

$1255 \mathrm{II}$.

$1256 \mathrm{II}$.

1257 II.

var.

var. " small..

var. Haydeni, Gabb.

var, is small...

var. Gabbana, Hemphill.

var. " small....

Section Mesodon.

GROUP OF H. GRISFOI.A.

I25S H. grisola, Pfr. Texas.

GROTP OF H. AIBOI,ABRIS.

I259 1I. Albolabris, Say. Mimuesota....... 1260 II. " var. Andrewsi, WV. A. Binn, Temmessee ........... 25

126I II. " var. major, Binn. Georgia.

1262 H. " var. exoleta, Say, Uhio..... I263 II. " var. thyroiles, say. Ohio. I264 H. multilineata, Say. Mlinnesota....

1265 II. Mitchelliana, Lea. Ohio........... I266 I1. Wheatleyi, Bld. North Carolina.. 126 \% H. l'enusylvanica, Green. Ohio..... I268 11. profunda, Say. Mimesota ......... 1269 H. Jejuma, Say. Floricla.

I2,0 II.

var.

GROIP OF H, PALLIATA.

127I 1I. palliata, Say. Tennessee........... I5

1272 I1. ". var. appressa, Say. X. C. I0

I273 H. . var. 
GROUP OF H, PALIIATA (Continned.) 1274 H. Romeri, Pfr. Texas I275 H. " " var. toothless............... I276 H. " var. Wetherbyi, Bld. Ten11. GROUP OF H. DEVIUS.

1277 H. Columbiana, var. labiosa,Gld. Ore. I278 H. " " var. " small. "

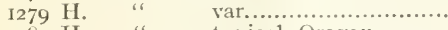
I2So H. “ " $\quad$ typical, Oregon..........

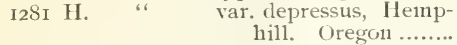
1282 H. " var.dentata, Hemphill. California............. $\mathrm{I}_{2} \mathrm{~S}_{3}$ H. " var. armigerus, Ancey. California..............

1284 H. devius, Gld, large. Washington.. I285 H. " var. cognatus, Hemphi1. Oregon ................ 1286 H. " var. Oregonensis, Hemphill. Oregon........ ${ }^{2} 87$ H. “ var. Blandi, Hemphill, large, Oregon........ I288 H. " var, Blandi, typical........ I289 H, “ var. binominata, Tryon. Idaho .................

$1290 \mathrm{H}$. “ var, binominata, medium. I29I H. "“ var. binominata, large. Iclaho .................

I292 H. "“ var. Mnllanii, Bld. Idaho. 1293 H. " var. Salnonensis, Tryon. Idalio ....

GROUP OF H. TRIDENTATA.

Umbilicus-open.

1294 H. tridentata, Say. sinall. Georgia. 1295 H. " var, large. Chio.......... I296 H. " " var. fallax, Say. Tenn.. I297 H. " var. Van Nostrandi, Bld. $1298 \mathrm{H}$. var. smill.

I299 H. I300 $\mathrm{H}$. Georgia.

I3OI II.

$1302 \mathrm{H}$.

I3O3 II. var. introferens, Bld. Tenn var. Sanbnrni, IV.G. Biıı. Idaho..................
var. Sanburni, small. Idaho.

var. Hopetonensis, Shut..... Florida.

var. Hopetonensis, sninall. Umbilicus-closed.

I304 H. tridentata, var. inflecta, Say. Ohio $1305 \mathrm{H}$. $1306 \mathrm{H}$. I307 H. var. " sniall. "
var. Ruglei, Sliutt, Ga.
var.

GROUP OF H. SPINOSUM.

r3o8 H. spinosum. Lea. Tennessee..

I309 H. barbigermm, Redf. Georgia....... GROUP OF H. STENOTREMA.

IzIo 11. Stenotrema, Fer. Tennessee ...... I3II 11 , $1312 \mathrm{H}$. var. small. " var, hirsutü.

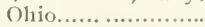
var. swall $\ldots \ldots \ldots \ldots \ldots$ var, " depresserl. Ohio
GROUP OF H, MONODON,

to 20 I3I5 H. monodon, Rackett, Minnesota..

20 I3I6 H. germa1mm, Gld. WVashington..... I3I7 H. ." var. small.

I3I8 H. loricata, G1d, large. Califormia... I3I9 H. " v var, nedium.

I5 1320 H. " " var, very small, “

15 I32I H. “ var. mmbilicata,

IO GEOUP OF H. TOWNSENDIANA.

I322 H. Townsenliana, var. ptychophora.

I5 Brown, small.......\$ I5

I5 $1323 \mathrm{H}$. $\quad$ var. ptychophora,

I5 large, Idaho..

I324 H. " var. ptychophora, albino. Idaho

20 I325 H. .. var, ptychophora, medium, Idaho.

15. $1326 \mathrm{H}$. var. ptychophora, typical. Idaho....

I5 $1327 \mathrm{H}$. $1328 \mathrm{H}$. var. castaneus.Idaho var. large. Idaho.....

I5 I329 H. Townsendiana, Lea, typicalWash.. I330 H. arrosa, var, exarata Pfr. Cal. I33I H, " var. " " elevated. "

I5 I332 H, “ var, " I333 H. " Glid. typical. "

Io I $334 \mathrm{H}$, " var. small, " 20 $1335 \mathrm{H}$. " var, flavus. " 20

I5 I336 H. " var, niger. " 20

I5 1337 II, " var. lepressed. " 20 I338 H. tudiculata, var. depressed, large. " 20

20 I 339 II. " " typical, " I5 $1340 \mathrm{H}$. " " var.subdolus, IIemphill I34I H. " var.subdolus, smail. I342 H, “ var. Tularensis, Hemphill. California.....

GROUP OF H. CALIFORNIENSIS,

I343 H, Californiensis, var. Diabloensis, I344 H. J. G. Cooper ......... vat. ramentoso, Gld. California............. var. Brillgesi, Newc. California ............ var. Bridgesi, sı11all. California ............ var.Nickliniana. Lea Califorriia............. var. Nickliniana, small.

I348 II. I349 H. Californiensis, Lea. typical, Calif.. 20 I350 11 . small

GROVP OH: H, FIDEIIS.

I35I II. fidelis, Gray. Oregon............... I5 I352 11. " var, minor, Bin11. Oregon. I353 11. " v var, dark.

1354 11. " var. subangulata. Hemphill I355 11. “ var. “ " small..... 1356 11. “ var. infunati, Crld........... 1357 11. “ var. "110r110num, Pfr. Cal. I358 11 . $1359 \mathrm{H}$. $1360 \mathrm{H}$ I36I H. I362 II. " var, sequoicola, Cooper.....
20 20 20 20 
1363 H. . var. Traskii, Newc., large.. 1364 H. " var. " I365 1 . $1366 \mathrm{H}$.

$1367 \mathrm{H}$. " var. cuyamacelısis ........... " var, ". small ..... var. proles, Itempliill.........

1368 H. rufocineta, Newe. Cal............. $1369 \mathrm{H}$.

I370 $\mathrm{H}$.

$137 \mathrm{I} \mathrm{H}$.

$1372 \mathrm{H}$.

$1373 \mathrm{H}$.

$\mathrm{I} 374 \mathrm{H}$.

I375 $\mathrm{H}$.

I376 11 .

$1377 \mathrm{H}$.

var. minor.....................

var. (rabl)i, Newe, large.

$\begin{array}{cc}\text { var. " } & \text { small ............. } \\ \text { var. } & \text { ". }\end{array}$

var.

var, facta, Newc...........

var. " elevated.......

var. " lepressed ....

var. ". bandless .....
GROUP OF H. KHILET'TI.

${ }_{37} \mathrm{~S}$ H. Kelletti, Fus. Califormia Islauts... 1379 H. " var. A, castaneus, Hemphill California Islanils..

I3tio II. var. niticlus, Hewplisll. California Islands.. I3SI H. ${ }_{13} \mathrm{~S}_{2} \mathrm{H}$. var. multilineata, Hemphill. Cal. Islands... var, frater, Hempliill. California Islands..

$\mathrm{I}_{3} \mathrm{~S}_{3} \mathrm{H}$.

${ }_{13} \mathrm{~S}_{4} \mathrm{H}$. var. Califormica, Hempbill. Cal. Islanils. var. Forbesi, Hemplill. California Islands.

$13 \mathrm{~S}_{5} 11$. " var. bicolor, Hemphill. Califormia Islands.:

I3 $86 \mathrm{H}$. " var, tricolor, Hempliill.

var. Stearnsiana, Gabl).

${ }_{13} \mathrm{~S}_{7} \mathrm{H}$.

${ }_{13} \mathrm{SS} \mathrm{H}$.

${ }_{13} \mathrm{~S}_{9} \mathrm{H}$.

I390 H.

I391 II.

$1392 \mathrm{H}$.

var. California............

var. sinall... islands. $1393 \mathrm{H}$.

20 20 20 20 20 20 20 15

15
15 15

15 15 15

15

15 423 P. Sterri Voith. Ttah.......

1.124 I'. Plandi, Morse. ['talı................

$10 \quad 1425$ P. Rowelli, Newc, California.......... Io

$1+26$ P. Californica, Rowell. California... 5

$201427 \mathrm{H}$. "f var. meriolionalis, Sterki. California ............ 15

201428 I. " " var. trinotata, Sterki. Cal. 1429 l". "“ var, catalinaria, Sterki."

$20 \quad 1430 \mathrm{P}$. corpulenta, Morse. Ctah ............ I 431 J'. castanea, Sterki. California.......

201432 P'. calamitosa, Pilsbry.

1433 P. Hemphilli, Sterki.
26

20

20

var. redimita, Binn. Islands

var. hybrida, Hempliill.

var. B.castanens, Heniphili aspersa, Mull., introduced. (a)ifornia

GROUP OF H. INTERCISA.

1394 II. Ayresiana, Newc. Cal. Islands ...

1396 H. intercisa, Bim. California Islands.

1397 H. " var. minor.

i39s H. " var. elegans.

1399 H. " " var. nepos.

$1400 \mathrm{H}$. " var. albicla.

1401 H. Tryonii, Newe. large.

1402 H. " " var. sniall.

$1403 \mathrm{H}$.

$1404 \mathrm{II}$.

$1405 \mathrm{H}$.

$1406 \mathrm{H}$.

$1407 \mathrm{H}$.

$140 \mathrm{H}$.

$1409 \mathrm{H}$.

$1410 \mathrm{H}$.

I4I I H.
I395 H. " var. " small.
I 4 I $2 \mathrm{H}$.

$1413 \mathrm{H}$.

var. varius

var. "6 A sniall.

var. nebulosa

var. " $\mathrm{B}$ sinall........

var. " C ..............

var. fasciata.

var. " D siswall .......

var. Californica ...........

var. "E $\mathrm{E}$ small...

var, albicla..................

var. "F sillall.

GROUP OF H. AREOLATA.

14I4 H. areolata, Fbs. Lower Califormia.. $1415 \mathrm{If}$.
Section Lucocheila.

1434 P. molica, Gld. Florida ............... 5

1435 P. Arizonensis, Gabb. Nevada........ 20

1436 P. rupicola, Say, Florida............... 5

1437 P. hordeacella, Pilsbry. Florida...... I0
FAMIIY STENOGYRID.F. GFives RUMIXA.

1445 R. subula, Pfr. South Carolina........ Section Melumella.

1446 R. gracillina, Pfr. Florida. GENI'S FERI:SSACIA

1447 F. subeylindrica, Lim. Etah, N. C... FAMHL CYLINDRELLIDF. GENUS CYLINDRFILA.

$144^{S}$ C. Poeyana, Pfr. Floricla. GENLS MACROCERAMUS.

I4t9 MI, poutificus, Gld. Florida. $145^{\circ} \mathrm{MI}$.

var. small.

\section{FAMIIL BLLIMILLIDA.}

\section{GENUS BULIMLUS.}

I45I B, multilineatus, Say. Florida.

1452 B. Marielinus, Poey. Florida. 
FAMILY ORTHALICIDA. GENUS LIGUUS.

I455 L. fasciatus, Muller. Florida.......... I456 L. " " var. solida, Say. Florida $1457 \mathrm{~L}$. I $45^{8} \mathrm{I}_{4}$ var. black banded. "“ var. variable.

GENUS ORTHALICUS.

1459 O. undatus, Brug. Florida FAMLY SUCCINIDE.

GENUS SUCCINEA,

I46o S. Haydenii, W. G. Binn. Utah ...... I46I S. Sillimani, Bld. California..

I 462 S. versicolor, Tryon. Lonsiana .......... 1463 S. Salleanus, Pfr.

I 464 S. Nuttalliana, Lea. Washington...... I 465 S. rusticana, Gld. California........... $1466 \mathrm{~S}$. Oregonensis, Lea. 467 S. obliqua, Say, Mimuesota .......... I 468 S. campestris, Say. Florida............ I 469 S. luteola, Gld. Texas................... I470 S. lineata, W. G. Biun. Utali.............. I47I S. avara, Say. California, Minn...... 1472 S. Gabbi, Tryon. Utah.................. I $473 \mathrm{~S}$.

Lower California
FAMILY VERONICELLIDE.

GENUS VERONICEL,I,A.

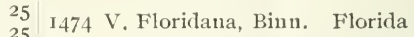

FAMILY ONCHIDIIDA.

GENUS ONCHIDIUN.

I475 O. Carpenteri, W, G. Binn, L. Cal...

25

\section{ORDER OPERCULATA.}

FAMILY CYCLOSTOMIDA.

GENUS CHONDROPOMA.

I476 C. dentatum, Say. Florida............ I5

$1477 \mathrm{C}$. " "

$1478 \mathrm{C}$. " var. " $\ldots \ldots \ldots \ldots . . . . .15$

FAMILY HELICINIDA.

GE,NUS HELICINA.

10 I479 H. orbiculata, Say. Texas....

5 I480 $\mathrm{H}$. " " var. " $\ldots \ldots \ldots \ldots . . .$. IO

IO $148 \mathrm{I}$ H. " " var. "

I5 $1482 \mathrm{H}$. * var. Hanleyana. La... Io

\section{FRESH-WATER SHELLS.}

\section{FAMILY RISSOIDA.}

GFNIS HSDROBIA.

\section{FAMILY STREPOMATID.E.}

\section{GENUS ANGITREMA.}

I483 A. verrucosa, Raf. Tennessee.

\section{Subgenus Pleurocera.}

I484 P. canaliculata, Say. Ohio... I486 P. affine, Lea.

1457 P. Henryanum, Lea. Georgia............ 74 S8 P. prasinatum, Conr. Alaban1a ...... I4S9 P. subulare, Lea. Minnesota.

\section{GFNUS GONIOBASIS.}

I 490 G. lepida, Lea, Alabama.

I 49 I G. castaneus, Lea. Tennessee......... 1492 G. proxima, Say. North Carolina.... 1493 G. plicifera, Lea. Oregon ............. 1494 G. " var. Oregonensis, Tryon.Ore $1495 \mathrm{G}$.

$1496 \mathrm{G}$. I497 G. I $498 \mathrm{G}$. I499 G. I $500 \mathrm{G}$. I5OI G. I5O2 G. $1403 \mathrm{G}$.

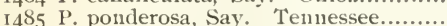

var. bulimoides, "“ " var. I,eai, Hemphill.. " " var. passing into tlie next " var. rudens, Rve.

var. silicnla, Gld. var. circumlineata, Tryon" var. var. nigrina, Lea. var. Draytonii. Lea.

GFNUS ANCULOSA.
10 I506 H. egena? Gle. California

GENUS BITHYNELIA.

1507 B, turbiniformis, Tryon. California.

$10 \quad 1508$ B. Binneyi, Tryon. California......... $101509 \mathrm{~B}$.

Io GE,NUS AMNICOLA.

5 Io A. longinqua, Glil. Utah...

512 A pallida, Hald. New York...........

GENIT' SOMATOGYRUS,

5 I513 S. isogonns, Say. New York

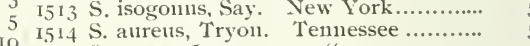

I5I5 S. parva, Lea.

I 5

GFiNUS FIUMINICOLA.

I5I6 F. fusca, Hald, Utah............... Io

Io I5I7 F. Hindsi, Baird. Oregon ............. Io

10 I5 \& F. Nuttalli, Lea. $\quad$ “ $\quad$............... 5

10 \$I9 F. " var. " ............ 5

IO GENUS POMATIOPSIS:

10 I520 P. lapidaria, Say, Minnesota.......... 5

$10 \quad 1521$ P. intermedia, Tryon. Nevada........ 5

10
10

\section{FAMILV VALVATIDAE.}

GFNUS VALVATA.

$1522 \mathrm{~V}$. tricarinata, Say. Minnesota

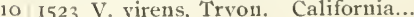

$101523 \mathrm{~V}$. virens, Tryoll. Califor

ro 1524 V. sincera, Say. Irlaho.

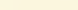




\section{FAMILY PALUDINIDAE.}

GENUS MEXANTIO.

I525 M. ponderosa, Say. Tennessee.

I526 M. integra, Say. Minmesota.

\section{FAMILY AMPULARII)A: \\ GENUS A MPIIARIA. \\ Subgenus Pomus.}

I527 P. caliginosus, Rve. Florida...

\section{FAMII, LIMNAIIJ)A:. \\ GENUS IIMN.EA.}

1528 L. stagnalis, Limm, Minnesota.

I529 L. “ var. appressa, Say. Utalı...

I530 L. " var. occilentalis, Hemphill.

I531 L. columella, Say. Georgia...

$$
\text { GENUS RADIX. }
$$

I532 R. ampla, Mighels, Itaho...

$\begin{array}{lll}\text { I533 R. " } & \text { var. } \\ \text { I534 R. " var. } & \text { Californial }\end{array}$

GENUS I,JMNOPHYSA.

I535 L. catascopium, Say. Oregon $1536 \mathrm{~L}$. " var.
1537 L. $1538 \mathrm{~L}$. " $\quad$ var. bulimoides? I,ea. Italı.. I539 L. " var. passing into the next... 1540 I. caperata, Say. IVashington...

I54I I. " var. Minnesotá 1542 L. " typical, Utah................. 1543 L. Inumilis, Say. Oregon.

I544 L, proxima, Lea. California.............. I545 L. desidiosa, Say, Italı..

I546 I.

$1547 \mathrm{~L}$.

I548 L.

var.

var.

IVaslington

Wyoning

I 549 L. palustris, var. Nuttalliana, J,ea. Etah

$1550 \mathrm{I}_{1}$ " " var. C'al.

I55I J. ", var. Idalio

$155^{2}$ I. $\therefore$ var. urmbrosa, Say, Etah.

1553 L. " var. "ial

I554 L. " " var. clorles, Say. Itah.

$1555 \mathrm{I}$. "

I556 L. " var. expansa, Hald. Cal.

1557 L. " " var, reflexa, Say. Miun.

155 L. " " var. " Say.

1559 I. " " var. " Say.

1560 L. “ var. attemuata.

1561 L. “ var. Zebra, Tryou.

1562 I. " var. Kirtlandiana, I,ea. Ltalı.

GFNUS PHYSA.

1563 P, Gabbi, Tryon. Califormia, ()regon 1564 P. Carltomii, Lea. Califormia ............

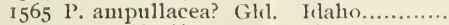
I 566 P. Sayi? Tappen. Utalı.

1567 P. gyrina, Say. Califormia .............. I56S P. " small, V'ashington.......... I569 P. lieterostropha, Say. Oregon, Etalı. $1570 \mathrm{P}$. " var. malleata, Tryon. C'al. 1571 P. " var, felsegera, Lea.

1572 P. “ var, penicillata, Hemplitl. Califormia..............

1573 P. “ var, Traskii, Tryon, Cal..

1574 P. " var, virginia, Gld. "...

1575 P. " var. occidentalis, Tryon.Cal.

I576 P. " var, elliptica, J,ea. Wasli'n

GENUS PHYSA. (Continued.)

1577 P. heterostroplia, var. Warreniana,

IC

(1) ${ }^{1} 57 \mathrm{~S} \mathrm{P}^{3}$.

$1579 \mathrm{I}$.

15 so $P$,

$15 \mathrm{Si}$

${ }_{15} \mathrm{~S}_{2} \mathrm{I}^{\prime}$.

${ }_{15} \mathrm{~S}_{3} \mathrm{I}^{\prime}$

$15 \$ 4$ P. integra, Hald. California and La... $15 \$ 5 \mathrm{P}$. “ var.

$1515 \mathrm{S6} \mathrm{P}$. Cooperi, I,ea.

15 is $87 \mathrm{P}$. costata, Newc.

I59l P. colnmbiana, Hemplill, Oregon...

SIBFAMILY POMPHOLIGINA:

5

5

Whorls subcarinated above and below. I596 I'. trivolvis, var. ammon, Gld. Calif... 25 1597 P. “ var. Traskii, Lea. Wash..... 25 I59S P. " "var, occidentalis, Cooper, Or. 20 1599 P. “ var. passing into next, Ten.. I5 $1600 \mathrm{P}$. " " var. bicarinatus, Say. Ore... IO I60I P. " var. " large flat, Min. Io IVhorls roumled above, subcarinated beneath. Lip of the apeature rising above the plane of the spire.

602 I'. trivolvis, var. Minnesota 10

Iips even with or fall the spire.

I607 P. trivolvis, var. lentus, Say, Mimu... I0 I6os P. " var. flatter, "... Io 1609 I'. " var. fallax, Texas........... 10) 1610 P.. var. near glabratus, Calif... in to 1611 P. ". var. near tumichs, Wash... I 10) 1612 P. campanulatus, Say. Minmesota..... I613 I'. J,iehmani, I)mker, Texas............ 1014 P'. Havenensis, Pfr, Lousiana......... 51615 P. allus, Mull. Mimmesota.

\section{Subgenus Grranlus.}

5616 (i. vermiculatus, Gld. Califormia...... Io

16 I (i. parvus, Say. 
GENUS SEGMENTINA.

1622 S. armigerus, Say. Minnesota......... S UBFA MII, Y A NCY L I N A GENUS ANCYLUS.

1623 A. fuscus, Hald. Louisiana............ I624 A. Haldemani, Lea. Tennessee........ I625 A. subrotundatus, Tryon, Oregon.... I626 A. Kootaniensis, Baird, Washington.. 1627 A. paralellus, Hald. Minnesota....... 1628 A. " var. fragilis, Tryon, Ore..... I629 A. rivularis, Say. Miunesota.......... Subgenus Acroluxus.

I630 A. Nuttalli, Hald. Oregon............. GENUS GUNDLACHIA.

1631 G. Californica, Ro vell, (young) Cal...

\section{CLASS PELECYPODA.}

\section{FAMILY CYRENIDA.}

GENUS SPHARIM,

1632 S. sulcatum, Lan1. Minnesota.

1633 S. occidentale, Prime. Idaho...........

I634 S. roseum, Prime, Washington.......

I635 S, stramineum, Conr. California......
GENUS SPHERUM. (Continued.)

51636 S. rhomboideum, Say. Idaho........ 1637 S. truncatum, Linsley. Minnesota... 1638 S. solidulum, Prine. . " . ... 1639 S. patella, Gld. 1640 S. triangulare ? Say. Louisiana................... 164 I S. lenticula, Gld. Washington.......... GENUS PISIDIUM.

1642 P. compressum, Prime. Utah......... I643 P. ferruguneum? Prime. Washington. 1644 P. nltranontamum, Prime. Utah..... 1645 P. occidentalis, Newe. California..... 1646 P. abditum, Hald.

Io 1647 P. equilaterale, Prime, New York... I64S P. consanguinemu? Prime. Nevada..

\section{FAMILY INIONIDAE.}

GENUS MARGARITANA.

I649 M. falcata, Gld, purple, Idaho........ 1650 M. "var, White, " ....... 20 GENUS ANODONTA.

165 I A. Walılamatensis, Lea. California.. 20 51652 A, Nuttalliana, Lea. California........ 20 5 I653 A. Oregonensis, Lea. Oregon......... 20 5 I654 A. Californiensis ? Lea. Idaho......... 20 5 . 655 A. Angulata, Lea. Cal. and Idaho... 20

\section{FOSSIL SHELLS}

\section{From the Later Teritary Beds of Upper and Lower California.}

1656 Laqueus Californicus, Dall.

I657 Cryptomya California, Conr.

Cryptom

1658 Corbula, lnteola, Cpr.......................

I659 Solecurtus Californianus, Conr.

I660 Solen rosaceus, Cpr.

1661 Macoma Secta, Conr.

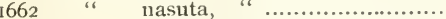

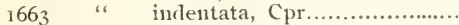

1664 " sp.

I 665 Tellina Bodgensis, Hds...................

I666 " " punicea, var.........................

r667 " " tumida, Sby....

1668 ، sp............

r669 Angulus Gouldi, Cpr..............................

I670 Clementia subdiaphana, Cpr...............

167 r Anniantis callosa, Cinr......................

1672 Tivela crassatelloides, Conr...

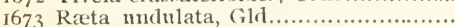

I674 Chione simillima, Sby..........

I675 " succincta, Val..

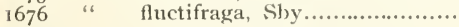

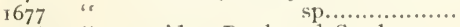

1678 " guidea, Brod. and Sowb..........

I679 Dosinia ponderosa, Gray.................

I680 Tapes staminea, Conr.

168 I Saxidomus Nuttalli, Conr.
$168_{2}$ Petricola pholadiformis, var....

$168_{3}$ Cardium procerum, Sowb.

1684 Liocardinm, Conr.

I685 Cardita ventricosa, Gld.

I686 Lucina nuttalli, Conr...

1687 " crenulata, Cpr.

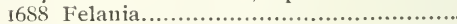

I689 Arca tuberculusa, Shy ....................

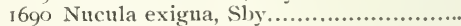

1691 Leda.

1692 Pecten C'errosensis, Gabb.

1693 " expansus, Dall

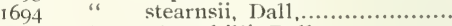

1695 " " Hemphilli, Dall................

1696 " ، iequisulcatus, Cpr.................

I697 “ " paucicostatus, Cpr..............

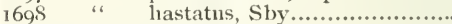

1699 Janaira dentula, Sby..

I700 Ostrea lurida, Cpr...

I701 " amara, Cpr.

1702 " Cerrosensis, Gabb.

I 703 Tornatina culcitella, Gld.

1704 "“ inculta, Gld...........

I705 Melampus olivaceus, Cpr.

I706 Dentalum liexagonum, Sby. 
I707 Dentalum semipolitum, Cpr.

I 708 A. insessa, Hds.

I 709 Fissurella volcano, Rve

I710 Chlorostoma Pfeifferi, Phil

1711 " aureotinctum, Fbs

1712 " funebrale, Ad.

I713 Calliostoma Palmeri, Dall.

I714 " tricolor, Gabb

I7I5 Omphalius fuscescens, Phil

1716 Crucibulum spinosum, Sby.

1717 Crepidula adunca, Sby

1718 " rugosa, Nutt..

1719 Serpulorbis squamigerus, Cpr...........

1720 Turritella gonostoma, var................

1721 " Cooperi, Cpr.

1722 Cocum Californicum, Dall.

1723 Cerithidea Sacrata, Glil.

1724 " " var. allonodosa.....

1725 Cerithuim Stercus-muscarum...

1726 Bittium quadrifilatum, Cpr.

1727 Lacuna vincta Mont. Cpr.

1728 Myurella Simplex, Cpr.

1729 Drillia inermis, Hds..

I730 " Hemphilli, Stearus

1731 Pleurotoma maculata, Sby.

I732 Sercula Carpenteriana, Gabb.

1733 Mangilia angulata, Cpr...

1734 Conus Californicus, Hds.

I735 Eulima micans, Cpr...
1736 Scalaria Hinılsii ? Cpr...

1737 " Hemphilli, Dall

$173^{8} \quad$ “ iurlianorum, var, tincta

1739 Opalia varicostata, Stearns

1740
I741 Neverita Recluziana, Petit.

I742 Ranella nuriciformis, Brod.

1743 ." Califormica, H.ls...

1744 Oliva arenosa, Iam.

1745 "angulata, I,an1...

1746 Olivella biplicata, Cpr.

1747 . bietica, Cpr....

1748 Nassa fossata, Glil.............

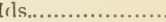

$175^{\circ}$ " tegula, Rve.

I751 Alia gansapata, Gld

1752 " Gonlii, Cpr.

1753 Anaclis coronata, Sby.

1754 Amphissa versicolor, I)all.

I755 Strombiua gibberula, Sby

1756 Monoceros engonatum, Conr.

I757 Chorus Belcheri, Hds.

1758 Cerostoma monoceros, Sowb.

1759 Siphonalia pallida, B and S.

1760 Pteronotus festivus, Hds.

I76I Murex radix, Gmel.

I 762 Balanus,

I763 E,china,

sp

sp 



\section{PRICES OF COLLECTIONS.}

\section{AMATEUR COLLECTIONS.}

Selected by myself and sent by mail, postage prepaiıl.

50 species, 100 specimens

83 on

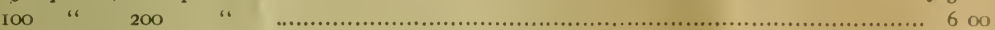

$150 \quad$ " $300 \quad$ "

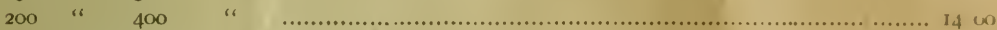

\section{REGULAR STUDENTS' COLLECTIONS.}

Selected by myself.

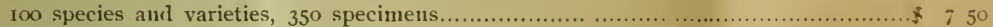

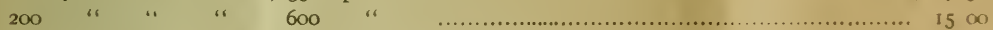

300 " " " " $\quad 850 \quad$ "

500 " " " " "

1000 " " " " " $3000 \quad$ " "

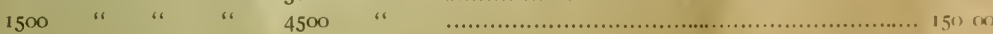

\section{TERTIARY FOSSILS.}

I00 species, 200 specinens

Special rates for Selecterl Collections.

I can supply nuany of the species en-suite that is from the very young to the arlult.

The prices in the colums on each page will be maintainerl for single specinens, but it disconnt of Io per cent. will be allowed on all orders of $\$ 5.00$ ant over.

The group of H. strigosa, numbers 1144 to $125 \%$, inclusive, will be sold as a wliole nuly: II 3 varieties, 226 specimens; price $\$ 15$.

Terms: Cash with order. Remittences ean be nuarle by l'ostoffice Money Oriler, ilraft, ete. (Personal checks not taken.)

Address,

HENRY HEMIPHILL.

P. O. Box 973, San Diego, California. I’. S. A. 


\section{FOR SALE.}

BOOIKS OH

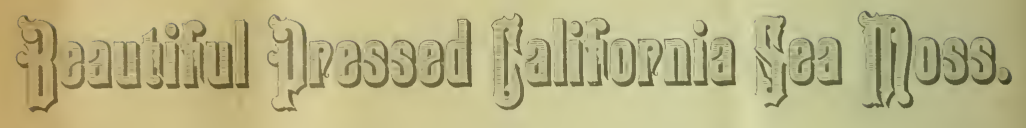

Size of Book, \& 1=2 x 11 mones,

With a handsome cover and containing eight artistically mounted specimens of the most beautiful California Sea Moss, on cardboard leaves, with a handsome border.

\section{SPECIMENS \& SPECIMENS.}

A Beautiful Present for a Lady.

PRice by Mail, Registered and PREPAid, Only \$1.25. Aclitress, 


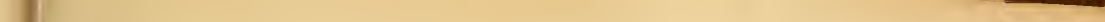


$Q$
$P=8$ 



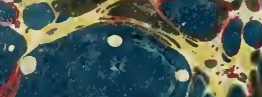

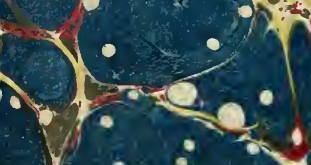
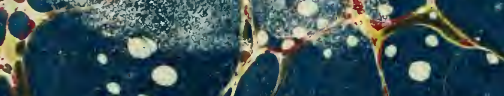

$x \cdot 0 x$

$\therefore \div$

$\therefore 0^{\circ}=0$

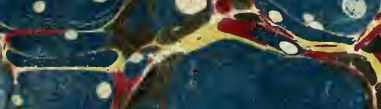

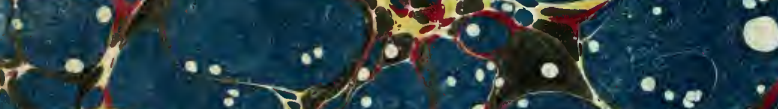

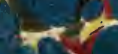

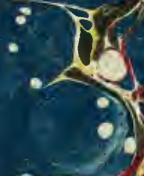

$0^{\circ}$ $\because \because 20$ 1 02.80 .2$.

$(8.5)$ 1. 3 2. 



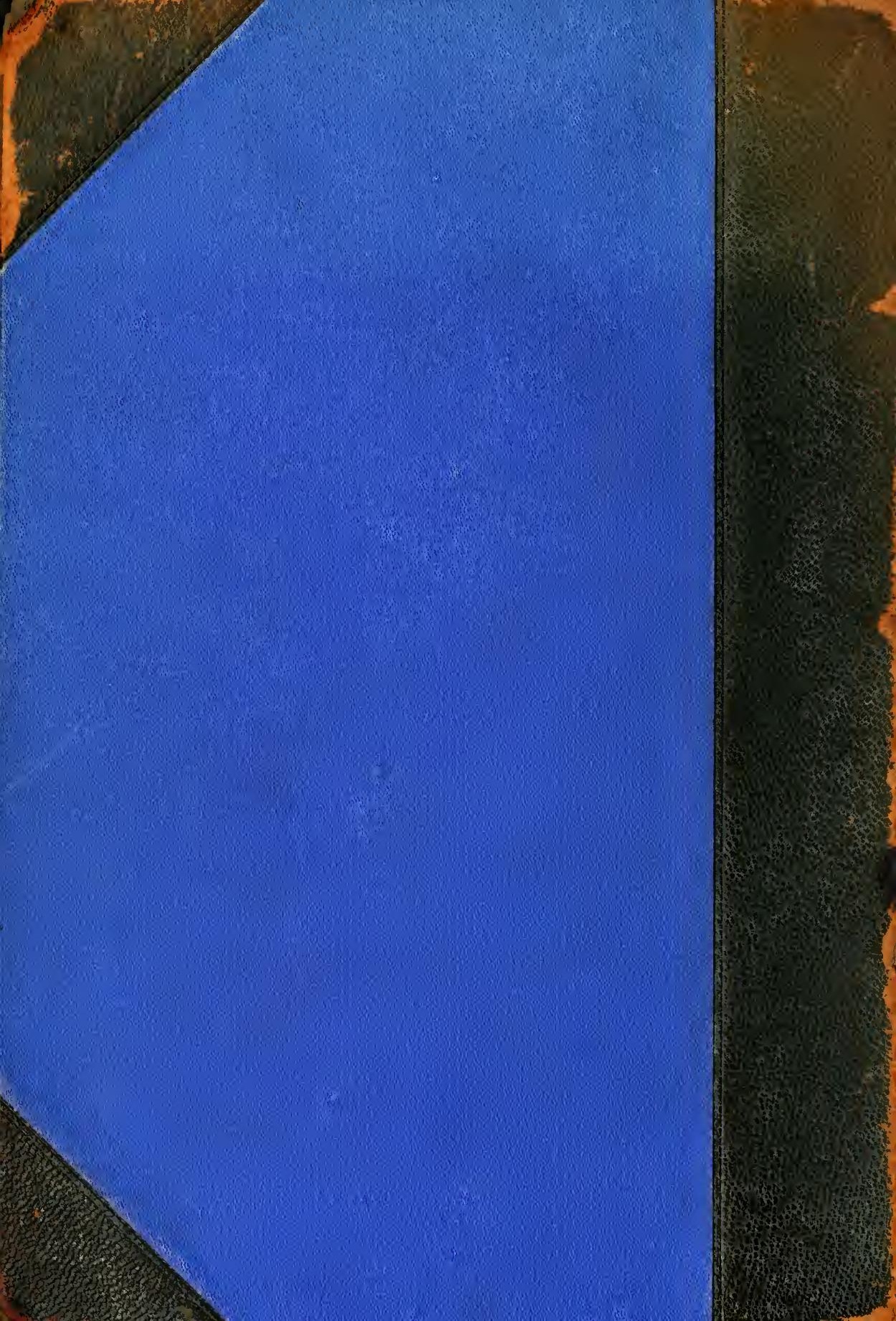

